\title{
EQUIVALENCES OF COISOTROPIC SUBMANIFOLDS
}

\author{
FLORIAN SCHÄTZ AND MARCO ZAMBON
}

\begin{abstract}
We study the role that Hamiltonian and symplectic diffeomorphisms play in the deformation problem of coisotropic submanifolds. We prove that the action by Hamiltonian diffeomorphisms corresponds to the gauge-action of the $L_{\infty}$-algebra of Oh and Park. Moreover we introduce the notion of extended gauge-equivalence and show that in the case of $\mathrm{Oh}$ and Park's $L_{\infty}$-algebra one recovers the action of symplectic isotopies on coisotropic submanifolds. Finally, we consider the transversally integrable case in detail.
\end{abstract}

\section{Contents}

Introduction

1. (Pre-)Symplectic Geometry

2. Deformations of coisotropic submanifolds

3. Hamiltonian diffeomorphisms

4. Symplectomorphisms

5. The transversally integrable case

Appendix A. Fibrewise entire Poisson structures

References

\section{INTRODUCTION}

Coisotropic submanifolds form an important class of sub-objects in symplectic and Poisson geometry. They naturally generalize Lagrangian submanifolds, play an important role in the theory of constraints and also appear in theoretical physics in the form of "branes", i.e. boundary conditions of sigma models [7, 1].

In this note we consider coisotropic deformations inside a symplectic manifold. The nearby deformations of a Lagrangian submanifold $L$ are well-understood: by Weinstein's normal form theorem, one can replace the ambient symplectic manifold by the cotangent bundle $T^{*} L$. The graph of a 1 -form $\alpha$ is Lagrangian if and only if $\alpha$ is closed. If one identifies closed 1-forms which are related through an Hamiltonian isotopy, one arrives at the first de Rham cohomology group $H^{1}(L, \mathbb{R})$ of $L$ as the appropriate moduli space of nearby Lagrangian deformations.

The generalization of these statements to coisotropic submanifolds is not obvious, since the space of coisotropic deformations is not linear and not even modelled on a topological vector space, see [20, 12]. However, the general pattern of deformation theory teaches us that

2010 Mathematics Subject Classification. 53D05, $16 \mathrm{E} 45$. 
every deformation problem ${ }^{1}$ should be captured by differential graded Lie algebras or their homotopical cousins, known as $L_{\infty}$-algebras. That this is indeed the case was established by $\mathrm{Oh}$ and Park in [12. To be more precise, $\mathrm{Oh}$ and Park constructed an $L_{\infty}$-algebra that controls the formal deformation problem for coisotropic submanifolds. In the special case of a Lagrangian submanifold $L$, their construction recovers the de Rham complex of $L$.

In [16], we studied convergence issues arising in the framework of [12. One finds that the Maurer-Cartan equation, which replaces the condition of being closed from the Lagrangian case, is always convergent, and that it converges to zero if and only if one is dealing with a coisotropic deformation 2

Having established a firmer link to actual geometric deformations, it is natural to turn attention to the geometric symmetries that are present in the problem. In particular, one might wonder how the actions of Hamiltonian and symplectic isotopies on the space of coisotropic deformations can be understood. A natural symmetry acting on MaurerCartan elements of $\mathrm{Oh}$ and Park's $L_{\infty}$-algebra are the inner automorphisms, known as gauge-transformations. Our main result is that these agree with the action by Hamiltonian isotopies, while the action by symplectic isotopies agrees with certain extended gaugeequivalences, which we specify below.

In Section 3 we deal with Hamiltonian isotopies. It turns out that the gauge-transformations of Oh and Park's $L_{\infty}$-algebra correspond to certain special Hamiltonian isotopies. The remaining problem is to show that any Hamiltonian isotopy can be reduced to such a special one. This is parallel to the Lagrangian situation: there the main task is also to show that an arbitrary Hamiltonian isotopy can be reduced to a function $f$ on the Lagrangian submanifold, which acts on the space of closed 1-forms (whose graphs we are interested in) simply by $\alpha \mapsto \alpha+d f$. We establish the appropriate generalization in Theorem 3.21, Subsection 3.5. As a consequence, we identify

$$
\frac{\{\text { coisotropic submanifolds }\}}{\text { Hamiltonian isotopies }} \cong \frac{\{\text { Maurer-Cartan elements }\}}{\text { gauge-equivalences }},
$$

which is the content of Theorem 3.22, For an alternative treatment within the BFVformalism we refer to the article [15] by the first named author.

Section 4 is concerned with symplectic isotopies. Given a Lagrangian submanifold, any of its Lagrangian deformations is related to the original submanifold by a symplectomorphism, so we do not obtain an interesting moduli space. In the general coisotropic case the situation is much more complicated and we do obtain another reasonable equivalence relation on the space of deformations by considering symplectic isotopies. In order to fit this into the algebraic framework, we review the construction of Oh and Park's $L_{\infty}$-algebra [12] [2] using Voronov's derived bracket construction [18, 19].

We show that every $L_{\infty}$-algebra which arises through Voronov's construction comes along with additional automorphisms. As a consequence, we obtain more ways to identify Maurer-Cartan elements. We refer to this extended equivalence relation as extended gauge-equivalence. The content of Theorem 4.18, Subsection 4.4 is that if one applies this

\footnotetext{
$1 \ldots$ in characteristic zero...

${ }^{2}$ For an alternative treatment of the coisotropic deformation problem in terms of a Maurer-Cartan equation, see [14.
} 
construction to Oh and Park's $L_{\infty}$-algebra, one precisely recovers the action of symplectic isotopies on the space of coisotropic deformations. As a consequence, we obtain the identification

$$
\frac{\{\text { coisotropic submanifolds }\}}{\text { symplectic isotopies }} \cong \frac{\{\text { Maurer-Cartan elements }\}}{\text { extended gauge-equivalences }}
$$

see Theorem 4.19.

In Section 5 , we consider coisotropic submanifolds which are transversally integrable. This regularity condition allows one to make some of the previous constructions more explicit. In particular, one can give a formula for nearby coisotropic deformations which are obtained by an Hamiltonian or symplectic isotopy from the original coisotropic submanifold, see Proposition 5.12.

In Appendix $\mathrm{A}$ we discuss the extension of our results to fibrewise entire Poisson structures. In [16 it was shown that the coisotropic deformation problem for those Poisson structures is also controlled by an $L_{\infty}$-algebra. Most of the results established in the bulk of the paper carry over to the case of fibrewise entire Poisson structures. We explain the necessary modifications in the appendix.

Organization of the paper: In Section 1 we recall background material on coisotropic submanifolds. In Section 2 we review the results about deformations of coisotropic submanifolds which are relevant in the subsequent discussion. In particular, we introduce $\mathrm{Oh}$ and Park's $L_{\infty}$-algebra and review the relation between its Maurer-Cartan elements and the deformation problem. In Section 3 we discuss Hamiltonian isotopies, while in Section 4 we deal with symplectic isotopies. In Section 5 , we consider the case of transversally integrable submanifolds. Finally, Appendix $\mathrm{A}$ describes the extension of our results to fibrewise entire Poisson structures.

Comparison with the literature: While we were completing this note, the preprint 10 by Lê, Oh, Tortorella and Vitagliano appeared. It considers coisotropic deformations in the very general setting of abstract Jacobi manifolds, which include Poisson and symplectic manifolds as special cases. There is an overlap between the results presented there in [10, Subsection 4.4] - once specialized to the symplectic case - and one of the main sections of the present note, namely Section 3. In particular, Thm. 3.21(i.e. the equivalence of Hamiltonian equivalence and gauge-equivalence, under a compactness assumption) corresponds to [10, Corollary 4.24]. Notice that in the latter the assumption on the compactness of the coisotropic submanifold is omitted.

Acknowledgements: M.Z. thanks Luca Vitagliano and Alberto Martín Zamora for useful conversations. In particular, the proof of Lemma 3.14 was communicated to us by Luca Vitagliano. Moreover we thank Hông Vân Lê for useful comments on a draft-version of this note. Last but not least we thank the referee for the helpful suggestions which improved the manuscript.

F.S. was supported by the Center of Excellence Grant "Centre for Quantum Geometry of Moduli Spaces" from the Danish National Research Foundation (DNRF95). M.Z. was 
partially supported by grants MTM2011-22612 and ICMAT Severo Ochoa SEV-2011-0087 (Spain), and Pesquisador Visitante Especial grant 88881.030367/2013-01 (CAPES/Brazil).

\section{1. (Pre-)Symplectic Geometry}

We summarize background information about coisotropic submanifolds and associated structures.

Remark 1.1. Throughout this paper, $(M, \omega)$ will denote a symplectic manifold. Let $C$ be a submanifold of $M$ and $E \rightarrow C$ a vector subbundle of $\left.T M\right|_{C}$. The symplectic orthogonal $E^{\perp}$ to $E$ is the vector bundle whose fibre over $x \in C$ is

$$
E_{x}^{\perp}:=\left\{e \in T_{x} M \text { such that } \forall v \in E_{x} \text { we have } \omega_{x}(e, v)=0\right\} .
$$

Another way to characterize $E^{\perp}$ is as the pre-image of the annihilator $E^{\circ}$ of $E$ under the sharp-map

$$
\omega^{\sharp}: T M \rightarrow T^{*} M, \quad v \mapsto \omega(v,-) .
$$

Definition 1.2. A submanifold $C$ of $(M, \omega)$ is coisotropic if the symplectic orthogonal $T C^{\perp}$ to $T C$ is contained in $T C$.

Remark 1.3. An alternative way to express the coisotropicity of $C$ is in terms of the Poisson bivector field $\Pi$ associated to $\omega$, defined by the requirement that $\Pi^{\sharp}: T^{*} M \rightarrow$ $T M, \quad \xi \mapsto \Pi(\xi,-)$ equals $-\left(\omega^{\sharp}\right)^{-1}$. Let $\mathcal{X}^{\bullet}(M)$ denote the space of multivector-fields on $M$, i.e. sections of $\wedge T M$. There is a natural projection map

$$
P: \chi^{\bullet}(M) \rightarrow \Gamma\left(\wedge\left(\left.T M\right|_{C} / T C\right)\right),
$$

which is given by restricting multivector-fields to $C$, followed by composition with the natural projection $\left.\wedge T M\right|_{C} \rightarrow \wedge\left(\left.T M\right|_{C} / T C\right)$. The submanifold $C$ is coisotropic if and only if the Poisson bivector field $\Pi$ lies in the kernel of $P$.

Definition 1.4. A two-form $\eta$ on $C$ that is closed and whose rank is constant is called a pre-symplectic structure.

Lemma 1.5. Let $C$ be a coisotropic submanifold of $(M, \omega)$. The pull back of $\omega$ to $C$ along the inclusion $\iota: C \hookrightarrow M$ is a closed two-form of constant rank $2 \operatorname{dim} C-\operatorname{dim} M$. We denote this pre-symplectic structure by $\omega_{C}$.

\section{Remark 1.6.}

(1) Let $\eta$ be any pre-symplectic structure on $C$. The closedness of $\eta$ implies that the kernel of $\eta^{\sharp}: T C \rightarrow T^{*} C$ is an involutive subbundle of $T C$. Hence $C$ is equipped with a foliation, called the characteristic foliation of $\eta$.

(2) We now consider the case of the pre-symplectic structure $\omega_{C}$ associated to a coisotropic submanifold $C$ of $(M, \omega)$. We always denote the kernel of $\omega_{C}$ by $K\left(=T C^{\perp}\right)$ and the corresponding characteristic foliation by $\mathfrak{F}$ in this situation. Moreover, observe that, in this situation, the vector bundle morphism

$$
\left.\left.T M\right|_{C} \stackrel{\omega^{\sharp}}{\longrightarrow} T^{*} M\right|_{C} \longrightarrow\left(T C^{\perp}\right)^{*}
$$

is surjective and has kernel $T C$. Hence we obtain an isomorphism between the normal bundle $\left.T M\right|_{C} / T C$ and $K^{*}$. 
Remark 1.7. We saw that every coisotropic submanifold comes along with a pre-symplectic structure. An important observation is that this can be reversed: every pre-symplectic structure can be realized as the pre-symplectic structure associated to a coisotropic submanifold. Moreover, this realization is essentially unique. We start with a pre-symplectic structure $\eta$ on a manifold $C$. Let $K$ be the kernel of $\eta^{\sharp}$ and $G$ a complement to $K$. The choice of $G$ yields an inclusion $j: K^{*} \hookrightarrow T^{*} C$. Recall that $T^{*} C$ carries a canonical symplectic structure $\omega_{T^{*} C}$. We now combine $\eta$ and $\omega_{T^{*} C}$ into the two-form

$$
\Omega:=\pi^{*} \omega_{C}+j^{*} \omega_{T *} .
$$

on $K^{*}$, where $\pi$ denotes the projection map $K^{*} \rightarrow C$.

The two-form $\Omega$ restricts to $\eta$ on $C$ and is symplectic on a tubular neighborhood $U$ of the zero section $C \subset K^{*}$. We refer to $(U, \Omega)$ as the local symplectic model associated to the the pre-symplectic manifold $(C, \eta)$.

The local symplectic model depends on the choice of complement $G$ to $K$, but choosing different complements will lead to local symplectic models which are symplectomorphic in neighborhoods of $C$, and one can choose a symplectomorphism that restricts to the identity on $C$. Hence we will speak of the local symplectic model of $(C, \eta)$.

The following theorem of Gotay [5] asserts that actually every symplectic manifold $(M, \omega)$ into which $C$ embeds as a coisotropic submanifold, such that $\omega_{C}=\eta$, looks like the local symplectic model in a neighborhood of $C$ :

Theorem 1.8 (Gotay [5]). Let $C$ be a coisotropic submanifold of a symplectic manifold $(M, \omega)$. There is a symplectomorphism $\psi$ between a tubular neighborhood of $C$ inside $M$ and a tubular neighborhood of $C$ inside its local symplectic model $(U, \Omega)$. Moreover, $\psi$ can be chosen such that the restriction of $\psi$ to $C$ is the identity.

Throughout the rest of the paper we fix a local symplectic model $(U, \Omega)$ of the coisotropic submanifold $C$. Since the local symplectic model is a neighborhood of the zero section in a vector bundle $E \rightarrow C$, it comes equipped with an embedding of the zero section $C$ in $U$, with coisotropic image, as well as with a surjective submersion $\pi: U \rightarrow C$. Recall that $E$ is isomorphic to $K^{*}$, the dual to the kernel of the pre-symplectic structure $\omega_{C}$. To avoid unnecessary confusion about signs, we also assume that $U$ was chosen invariant with respect to fibrewise multiplication by -1 .

Summarizing, the setting we assume in the rest of the paper is:

$(M, \omega)$ is a symplectic manifold,

$C$ is a coisotropic submanifolds with induced presymplectic form $\omega_{C}$, $(U, \Omega)$ is the local symplectic model, where $U$ is a neighborhood of the zero section in a vector bundle $E \rightarrow C$.

\section{Deformations of COISOTROPIC SUbMANifolds}

We set up the problem of deforming a given coisotropic submanifold and review some relevant results, setting the stage for the subsequent development. In particular, the precise relationship between the deformation problem and the $L_{\infty}$ [1]-algebra of Oh and Park [12, 16] is recalled. 
2.1. The deformation problem. It is natural to wonder how the "space of coisotropic submanifolds close to $C$ " looks like, i.e. we ask

Which deformations of $C$ are coisotropic submanifolds of $(U, \Omega)$ ?

Definition 2.1. The space of coisotropic sections of $U$ is

$$
\operatorname{Def}_{U}(C):=\{s \in \Gamma(U) \text { : the graph of } s \text { is coisotropic inside }(U, \Omega)\} \text {. }
$$

We now translate the above question into:

$$
\text { How can one describe the set } \operatorname{Def}_{U}(C) \text { ? }
$$

Theorem 2.9 in Subsection 2.3 provides an answer to this question.

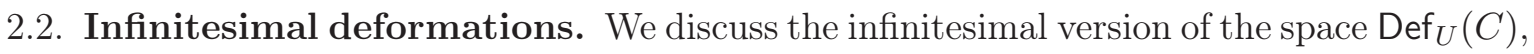
which turns out to be closely related to the foliated de Rham complex.

Remark 2.2. Recall from Section 1 that the kernel $K$ of the pre-symplectic structure $\omega_{C}$ on $C$ is involutive, and that the associated foliation $\mathfrak{F}$ of $C$ is called the characteristic foliation. One has the following foliated version of the de Rham complex:

$$
\begin{aligned}
\Omega_{\mathfrak{F}}(C):= & \Gamma\left(\wedge K^{*}\right), \\
\left(d_{\mathfrak{F}} \omega\right)\left(s_{0}, \ldots, s_{k}\right):= & \sum_{i=0}^{k}(-1)^{i} s_{i}\left(\omega\left(s_{0}, \ldots, s_{i-1}, \widehat{s_{i}}, s_{i+1}, \ldots s_{k}\right)\right. \\
& +\sum_{i<j}(-1)^{i+j} \omega\left(\left[s_{i}, s_{j}\right], s_{1}, \ldots, \widehat{s_{i}}, \ldots, \widehat{s_{j}}, \ldots s_{k}\right) .
\end{aligned}
$$

In Remark 1.6, we obtained a vector bundle isomorphism

$$
E=\left.T M\right|_{C} / T C \rightarrow K^{*}
$$

by restricting $\omega^{\sharp}$. This yields an isomorphism $\Gamma(\wedge E) \cong \Gamma\left(\wedge K^{*}\right)=\Omega_{\mathfrak{F}}(C)$. The foliated de Rham operator $d_{\mathfrak{F}}$ then corresponds to the operator

$$
\xi \mapsto P([\Pi, \xi]),
$$

where $\xi \in \Gamma(\wedge E)$ is interpreted as a vertical multivector-field that is constant along the fibres of $E$, and $[\cdot, \cdot]$ is the Schouten-Nijenhuis bracket, see [16, Proof of Prop 3.5] for more details.

Remark 2.3. We will show that the formal tangent space to $\operatorname{Def}_{U}(C)$ can be identified with the space of $d_{\mathfrak{F}}$-closed foliated one-forms on $C$. To this end, we rewrite the condition for a section $s$ of $U$ to be coisotropic in a more algebraic way. First, every section $s \in \Gamma(U)$ yields a diffeomorphism

$$
\psi_{-s}: E \rightarrow E, \quad(x, e) \mapsto\left(x, e-s_{x}\right),
$$

which maps $\operatorname{graph}(s)$ to the zero section $C \subset E$.

The graph of $s$ is coisotropic with respect to $\Omega$ if and only if the zero section is coisotropic with respect to $\left(\psi_{-s}\right)_{*} \Pi$, where $\Pi$ denotes the Poisson bivector field corresponding to $\Omega$. As discussed in Section 1, the latter statement can be expressed by saying that $\left(\psi_{-s}\right)_{*} \Pi$ lies in the kernel of the projection map

$$
P: \mathcal{X}^{\bullet}(E) \rightarrow \Gamma(\wedge E)
$$


given by restriction to $C$, composed with the projection $\left.\wedge T E\right|_{C} \rightarrow \wedge E$.

Hence, if we define $\mu$ to be the map

$$
\mu: \Gamma(U) \rightarrow \Gamma\left(\wedge^{2} E\right), \quad s \mapsto P\left(\left(\psi_{-s}\right)_{*} \Pi\right),
$$

a section $s$ will be coisotropic if and only if it is mapped to zero under $\mu$.

The map $\mu$ seems non-local since it involves the symplectic form away from $C$. However, the symplectic structure $\Omega$ of the local symplectic model $(U, \Omega)$ is determined by $\omega_{C}$. We will return to this point in Subsection 2.3, where we see that the equation $\mu(-s)=0$ can in fact be recovered as the Maurer-Cartan equation of an $L_{\infty}$-algebra whose structure maps are multi-differential operators on $C$.

Proposition 2.4. Let $s_{t}$ be a smooth one-parameter family of sections of $U$ which starts at the zero section $s_{0}=0$. Then

$$
\left.\frac{\partial}{\partial t}\right|_{t=0} \mu\left(s_{t}\right)=-d_{\mathfrak{F}}\left(\left.\frac{\partial}{\partial t}\right|_{t=0} s_{t}\right)
$$

under the identification $E \cong K^{*}$.

Proof. Consider the one-parameter family of diffeomorphisms $\psi_{-s_{t}}: E \rightarrow E$. The corresponding time-dependent vector field is $Y_{t}:=-\frac{\partial}{\partial t} s_{t}$, a vertical vector field which is constant on each fibre of $E$. Using this and the definition of $\mu$, we see that $\left.\frac{\partial}{\partial t}\right|_{t=0} \mu\left(s_{t}\right)$ equals the image under the projection $P: \chi^{\bullet}(E) \rightarrow \Gamma(\wedge E)$ of $\mathcal{L}_{\left.\frac{\partial}{\partial t}\right|_{t=0} s_{t}} \Pi=-\left[\Pi,\left.\frac{\partial}{\partial t}\right|_{t=0} s_{t}\right]$. By Remark 2.2 this is exactly the formula for the image of $\left.\frac{\partial}{\partial t}\right|_{t=0} s_{t}$ under $d_{\mathfrak{F}}$, if we apply the identification $E \cong K^{*}$.

Corollary 2.5. Let $s_{t}$ be a smooth one-parameter family of coisotropic sections of $E$ with $s_{0}=0$. Then $\left.\frac{\partial}{\partial t}\right|_{t=0} s_{t}$ is closed with respect to $d_{\mathfrak{F}}$.

Proof. We have $\mu\left(s_{t}\right)=0$ for all $t$ by Remark 2.3. hence the statement follows from Proposition 2.4

Remark 2.6. Proposition 2.4 identifies the space of closed elements of $\Omega_{\mathfrak{F}}^{1}(C)$ with the formal tangent space to $\operatorname{Def}_{U}(C)$ at $C$, where the formal tangent space is defined as the space of solutions to the linearized equation. We point out that it is known that not all cohomology classes of $H_{\mathfrak{F}}^{1}(C)$ can be realized through one-parameter families of deformations, see [20, 12].

2.3. Oh and Park's $L_{\infty}[1]$-algebra. We recall the $L_{\infty}[1]$-algebra associated to $C$ [12, 2] 3

Definition 2.7. An $L_{\infty}[1]$-algebra is a $\mathbb{Z}$-graded vector space $W$, equipped with a collection of graded symmetric brackets $\left(\lambda_{k}: W^{\otimes k} \longrightarrow W\right)_{k \geq 1}$ of degree 1 which satisfy a collection of quadratic relations [8], called higher Jacobi identities.

The Maurer-Cartan series of a degree zero element $\beta \in W$ is the infinite sum

$$
\operatorname{MC}(\beta):=\sum_{k \geq 1} \frac{1}{k !} \lambda_{k}\left(\beta^{\otimes k}\right) .
$$

\footnotetext{
${ }^{3}$ The reader is referred to [10, Appendix D] for a proof that the construction from [12] coincides with the one from [2], specialized to the symplectic case.
} 
We say that $\beta$ is a Maurer-Cartan element if its Maurer-Cartan series converges to zerd4. We denote the set of all Maurer-Cartan elements of $W$ by $\mathrm{MC}(W)$.

Remark 2.8. In order to describe the $L_{\infty}[1]$-algebra associated to the coisotropic submanifold $C$ of $(U, \Omega)$ as explicitly as possible, we consider the Poisson structure $\Pi$ associated to $\Omega$. As explained in Section 1 , the coisotropicity of $C$ is equivalent to $P(\Pi)=0$, where

$$
P: \chi^{\bullet}(E) \rightarrow \Gamma(\wedge E)
$$

is as in Equation (1).

As shown in [12] and [2, the space $\Gamma(\wedge E)[1]$ is equipped with a canonical $L_{\infty}[1]$-algebra structure. We denote the structure maps of this $L_{\infty}[1]$-algebra by

$$
\lambda_{k}: \Gamma(\wedge E)[1]^{\otimes k} \rightarrow \Gamma(\wedge E)[1] .
$$

The evaluation of $\lambda_{k}$ on $s \otimes \cdots \otimes s$ for $s \in \Gamma(E)$ yields

$$
\left.\lambda_{k}(s, \ldots, s):=P([[\ldots[\Pi, s], s] \ldots], s]\right),
$$

where $s$ is interpreted as a fibrewise constant vertical vector-field on $E$. Hence the MaurerCartan series of $s$ reads $\mathrm{MC}(s)=P\left(e^{[\cdot, s]} \Pi\right)$.

The following result, which is - partly in an implicit manner - contained in [12], is essentially [16, Thm. 2.8]. It relies on the fact that the Poisson bivector field associated to $\Omega$ is analytic in the fibre direction, which is true thanks to [16, Cor. 2.7]. In [16], such bivector fields are called fibrewise entire and most of the subsequent discussion carries over to such Poisson bivector fields. We refer the interested reader to Appendix A for more details.

Theorem 2.9. Consider the $L_{\infty}[1]$-algebra $\Gamma(\wedge E)[1]$ associated to the coisotropic submanifold $C$. For any $s \in \Gamma(E)$ such that $\operatorname{graph}(s)$ is contained in $(U, \Omega)$, the Maurer-Cartan series $\mathrm{MC}(-s)$ is pointwise convergent. Furthermore, for any such s the following two statements are equivalent:

(1) $\operatorname{graph}(s)$ is a coisotropic submanifold of $(U, \Omega)$.

(2) The Maurer-Cartan series $\mathrm{MC}(-s)$ converges to zero (in the sense of pointwise convergence).

Remark 2.10. In other words, if we restrict attention to those sections whose graphs lie inside $U$, the map $s \mapsto-s$ restricts to a bijection between the set of coisotropic sections

$$
\operatorname{Def}_{U}(C):=\{s \in \Gamma(U): \text { the graph of } s \text { is coisotropic inside }(U, \Omega)\}
$$

from Subsection 2.1, and

$$
\mathrm{MC}_{U}(\Gamma(\wedge E)[1]):=\{\text { Maurer-Cartan elements of } \Gamma(\wedge E)[1] \text { whose graphs lie in } U\} \text {. }
$$

Notice that the first structure map $\lambda_{1}$ of the $L_{\infty}[1]$-algebra $\Gamma(\wedge E)[1]$ coincides with the foliated de Rham differential $d_{\mathfrak{F}}$ under the isomorphism $\Gamma(\wedge E) \cong \Omega_{\mathfrak{F}}(C)$. We could a posteriori - use this fact to recover the infinitesimal description of $\operatorname{Def}_{U}(C)$ which we obtained in Subsection 2.2 .

\footnotetext{
4 ...with respect to a suitable topology. For the specific examples of $L_{\infty}[1]$-algebras with which we will be concerned later on, we will make this precise.
} 


\section{HAMiltonian DifFEOMORPHISMS}

In this section we investigate the action of Hamiltonian diffeomorphisms on the space of coisotropic submanifolds. More precisely, we provide a description of the induced equivalence relation on the space of coisotropic sections. As the main result, we show that for compact coisotropic submanifolds this equivalence relation coincides with the gaugeequivalence in $\mathrm{Oh}$ and Park's $L_{\infty}[1]$-algebra. This result was obtained independently by Lê, Oh, Tortorella and Vitagliano in [10, Corollary 4.24].

3.1. The deformation problem. Recall that by Definition 2.1 a section $s$ of $\pi: U \rightarrow C$ is called coisotropic if $\operatorname{graph}(s)$ is a coisotropic submanifold of $(U, \Omega)$, and that we denote the set of all such sections by $\operatorname{Def}_{U}(C)$.

Definition 3.1. Two coisotropic sections $s_{0}$ and $s_{1}$ are called Hamiltonian equivalent if there is a family of coisotropic sections $s_{t}$, agreeing with the given ones at $t=0$ and $t=1$, and an isotopy of Hamiltonian diffeomorphisms $\phi_{t}$ such that $\phi_{t}$ maps the graph of $s_{0}$ to the graph of $s_{t}$ for all $t \in[0,1]$.

Remark 3.2. To be more precise, we assume that we are given a locally defined Hamiltonian isotopy, i.e. a family of diffeomorphisms between open subsets of $U$, generated by a family of locally defined Hamiltonian vector fields, which maps graph $\left(s_{0}\right)$ onto $\operatorname{graph}\left(s_{t}\right)$.

It is straight-forward to check that Hamiltonian equivalence actually defines an equivalence relations on the set $\operatorname{Def}_{E}(C)$, which we denote by $\sim_{\text {Ham. }}$. We refer the interested reader to [15, Lemma 1] for a proof of this fact. It is natural to wonder about the equivalence classes of $\sim_{\mathrm{Ham}}$, so we define:

Definition 3.3. The Hamiltonian moduli space of coisotropic sections is the set

$$
\mathcal{M}_{U}^{\mathrm{Ham}}(C):=\operatorname{Def}_{U}(C) / \sim_{\mathrm{Ham}} .
$$

We ask:

$$
\text { How can one describe the set } \mathcal{M}_{U}^{\mathrm{Ham}}(C) \text { ? }
$$

Theorem 3.22 of Subsection 3.5 provides an answer in terms of the $L_{\infty}$ [1]-algebra of $\mathrm{Oh}$ and Park.

3.2. Infinitesimal moduli. We discuss the infinitesimal version of $\mathcal{M}_{U}^{\mathrm{Ham}}(C)$. In particular, we argue that the formal tangent space to $\mathcal{M}_{U}^{\mathrm{Ham}}(C)$ at the equivalence class of the zero-section $C$ is given by the first foliated cohomology $H_{\mathfrak{F}}^{1}(C)$, with $\mathfrak{F}$ the characteristic foliation of the pre-symplectic structure on $C$. The results of this subsection can be recovered - via specialization to the symplectic case - from the results obtained by Lê and Oh, 9, Subsection 6.3], who studied deformations of coisotropic submanifolds in locally conformal symplectic manifolds.

Remark 3.4. Let $\left(s_{t}\right)_{t \in[0,1]}$ be a family of coisotropic sections that starts at the zerosection. In Subsection 2.2 we saw that $\left.\frac{\partial s_{t}}{\partial t}\right|_{t=0} \in \Gamma(E)$ lies in the kernel of the complex $(\Gamma(\wedge E), P([\Pi,-]))$ and that the latter is isomorphic to the foliated de Rham complex $\left(\Omega_{\mathfrak{F}}(C), d_{\mathfrak{F}}\right)$. 
Proposition 3.5. Suppose that $\left(s_{t}\right)_{t \in[0,1]}$ is a family of coisotropic sections that starts at the zero-section and is trivial under Hamiltonian equivalence, i.e. there is an Hamiltonian isotopy $\phi_{t}$ such that the graph of $s_{t}$ coincides with the image of the zero section under $\phi_{t}$.

Then the cohomology class of $\left.\frac{\partial s_{t}}{\partial t}\right|_{t=0}$ in $H_{\mathfrak{F}}^{1}(C)$ is trivial.

Proof. Suppose that $\phi_{t}$ is generated by the family of Hamiltonian vector fields $X_{H_{t}}$. We can write $\left.\frac{\partial s_{t}}{\partial t}\right|_{t=0}$ as $P\left(X_{H_{0}}\right)=P\left(\left[\Pi, H_{0}\right]\right)$ (see Lemma 3.13 later on). We observe that the latter expression equals $P\left(\left[\Pi,\left.H_{0}\right|_{C}\right]\right)$, because $\left.\Pi^{\sharp}\right|_{C}$ maps the co-normal bundle to the tangent bundle $T C$, whose sections lie in the kernel of $P$. As a consequence, the cohomology class of $\left.\frac{\partial s_{t}}{\partial t}\right|_{t=0}$ equals the cohomology class of $P\left(\left[\Pi,\left.H_{0}\right|_{C}\right]\right)$, which is trivial. Now apply the isomorphism between $\Gamma(\wedge E)$ and the foliated de Rham complex from Remark 2.3.

Remark 3.6. For every $f \in \mathcal{C}^{\infty}(C)$, let $\phi_{t}$ be the flow of the Hamiltonian vector field $X_{\pi^{*} f}$, and $\left(s_{t}\right)_{t \in[0, \epsilon)}$ the family of coisotropic sections determined by $\operatorname{graph}\left(s_{t}\right)=\phi_{t}(C)$. Then the proof of Proposition 3.5 shows that $\left.\frac{\partial s_{t}}{\partial t}\right|_{t=0}$ corresponds to $d_{\mathfrak{F}} f$ under the isomorphism $\Gamma(E) \cong \Omega_{\mathfrak{F}}^{1}(C)$. Hence we can refine Proposition 3.5 as follows: the formal tangent space of the set of coisotropic sections which are trivial under Hamiltonian equivalence is precisely $\Omega_{\mathfrak{F} \text {,exact }}^{1}(C)$.

This and Remark 2.6 imply that the formal tangent space at zero to $\mathcal{M}_{U}^{\text {Ham }}(C)$ is $H_{\mathfrak{F}}^{1}(C)$. In the special case of $C$ Lagrangian, this reduces to the first de Rham cohomology $H^{1}(C)$ of $C$, as expected.

\subsection{Gauge-equivalence.}

Remark 3.7. Convergence issues aside, every $L_{\infty}[1]$-algebra $W$ comes along with a (singular) foliation on its set of Maurer-Cartan elements $\mathrm{MC}(W)$. On $W_{0}$, the elements of degree 0 , there is a distribution generated by vector fields $V_{\gamma}$ associated to elements $\gamma$ of degree -1 . At the point $\beta \in W_{0}$, the vector field $V_{\gamma}$ reads

$$
\lambda_{1}(\gamma)+\lambda_{2}(\gamma, \beta)+\frac{1}{2 !} \lambda_{3}(\gamma, \beta, \beta)+\frac{1}{3 !} \lambda_{4}(\gamma, \beta, \beta, \beta)+\cdots .
$$

The vector fields $V_{\gamma}$ are tangent to $\mathrm{MC}(W)$ and they form an involutive distribution there, hence we obtain a canonical equivalence relations on $\mathrm{MC}(W)$ :

Definition 3.8. Two Maurer-Cartan elements $\beta_{0}$ and $\beta_{1}$ of an $L_{\infty}$ [1]-algebra $W$ are gaugeequivalent if there is a one-parameter family $\gamma_{t}$ of degree -1 elements of $W$ and a oneparameter family $\beta_{t}$ of degree zero elements of $W$, agreeing with the given ones at $t=0$ and $t=1$, such that

$$
\frac{\partial}{\partial t} \beta_{t}=\lambda_{1}\left(\gamma_{t}\right)+\lambda_{2}\left(\gamma_{t}, \beta_{t}\right)+\frac{1}{2 !} \lambda_{3}\left(\gamma_{t}, \beta_{t}, \beta_{t}\right)+\frac{1}{3 !} \lambda_{4}\left(\gamma_{t}, \beta_{t}, \beta_{t}, \beta_{t}\right)+\ldots
$$

We presuppose that $W$ is equipped with a suitable topology and that the right-hand side of the above equation converges.

We apply this to the $L_{\infty}$ [1]-algebra structure on $\Gamma(\wedge E)[1]$ from Subsection 2.3. We are interested in $\mathrm{MC}_{U}(\Gamma(\wedge E)[1])$, the Maurer-Cartan elements of $\Gamma(\wedge E)[1]$ whose graphs lie in $U$ (see Remark 2.10). We define an equivalence relation on $\mathrm{MC}_{U}(\Gamma(\wedge E)[1])$ as in Def. 3.8, but additionally requiring that the one-parameter family of degree zero elements $\beta_{t}$ consists 
of sections of $U$ (rather than $E$ ). We use the bijection $\operatorname{Def}_{U}(C) \cong \mathrm{MC}_{U}(\Gamma(\wedge E)[1]), s \mapsto-s$ described in Remark 2.10 to transport the above equivalence relation to $\operatorname{Def}_{U}(C)$ :

Definition 3.9. Two coisotropic sections $s_{0}$ and $s_{1}$ are called gauge-equivalent, $s_{0} \sim_{\text {gauge }}$ $s_{1}$, if $-s_{0}$ and $-s_{1}$ are equivalent elements (in the sense above) of $\mathrm{MC}_{U}(\Gamma(\wedge E)[1])$.

Remark 3.10. We make the equivalence relation $\sim_{\text {gauge }}$ more explicit. Two elements $s_{0}$ and $s_{1}$ in $\operatorname{Def}_{U}(C)$ are declared gauge-equivalent if there is a smooth one-parameter family $s_{t}$ in $\Gamma(U)$, coinciding with $s_{0}$ and $s_{1}$ at the endpoints, such that

$$
\begin{aligned}
\frac{\partial}{\partial t}\left(-s_{t}\right) & =P\left(\left[\Pi, \pi^{*} f_{t}\right]\right)+P\left(\left[\left[\Pi, \pi^{*} f_{t}\right],-s_{t}\right]\right)+\frac{1}{2 !} P\left(\left[\left[\left[\Pi, \pi^{*} f_{t}\right],-s_{t}\right],-s_{t}\right]\right)+\cdots \\
& =P\left(e^{\left[\cdot,-s_{t}\right]} X_{\pi^{*} f_{t}}\right) .
\end{aligned}
$$

Here $-s_{t}$ is interpreted as a family of fibrewise constant vertical vector field and $f_{t}$ is a one-parameter family of smooth functions on $C$. Observe that the latter can be seen as a one-parameter family of degree -1 elements of the $L_{\infty}[1]$-algebra $\Gamma(\wedge E)[1]$. To rewrite the condition in more geometric terms, recall that for $s \in \Gamma(E), \psi_{s}$ is the diffeomorphism of $E$ that consists of fibrewise addition with $s$. Moreover, let $\mathrm{p}_{s}^{\mathrm{v}}$ be the projection of $\left.T E\right|_{\operatorname{graph}(s)}$ onto the vertical part of $T E$ along $T$ graph $(s)$.

We now compute

$$
\begin{aligned}
P\left(e^{\left[\cdot,-s_{t}\right]} X_{\pi^{*} f_{t}}\right) & =P\left(\left(\psi_{-s_{t}}\right)_{*} X_{\pi^{*} f_{t}}\right)=\mathrm{p}_{0}^{\mathrm{v}}\left(\left(\psi_{-s_{t}}\right)_{*}\left(X_{\pi^{*} f_{t}} \mid \operatorname{graph}\left(s_{t}\right)\right)\right) \\
& =\left(\psi_{-s_{t}}\right)_{*}\left(\mathrm{p}_{s_{t}}^{\mathrm{v}}\left(\left.X_{\pi^{*} f_{t}}\right|_{\operatorname{graph}\left(s_{t}\right)}\right)\right)=\mathrm{p}_{s_{t}}^{\mathrm{v}}\left(\left.X_{\pi^{*} f_{t}}\right|_{\operatorname{graph}\left(s_{t}\right)}\right) .
\end{aligned}
$$

We use [16, Prop. 1.15] in the first equality5, which applies since the vector field $X_{\pi^{*} f_{t}}$ is fibrewise entire in the terminology of [16]. In the last equality we used the fact that $\psi_{-s_{t}}$ maps $\operatorname{graph}\left(s_{t}\right)$ to the zero section $C$ and preserves the fibres of the projection $\pi: U \rightarrow C$.

After reversing the signs in front of $f_{t}$, this shows:

Proposition 3.11. Elements $s_{0}$ and $s_{1}$ of $\operatorname{Def}_{U}(C)$ are gauge-equivalent if and only if there is a one-parameter family $s_{t} \in \Gamma(U)$, agreeing with $s_{0}$ and $s_{1}$ at the endpoints, and $a$ one-parameter family $f_{t} \in \mathcal{C}^{\infty}(C)$ such that

$$
\frac{\partial}{\partial t} s_{t}=\mathrm{p}_{s_{t}}^{\mathrm{v}}\left(\left.X_{\pi^{*} f_{t}}\right|_{\operatorname{graph}\left(s_{t}\right)}\right)
$$

holds for all $t \in[0,1]$.

3.4. Technical Lemmata. We establish some technical lemmata that we use subsequently to relate various notions of equivalence between coisotropic sections.

Remark 3.12. Throughout this subsection, $A$ denotes a vector bundle over a smooth manifold $M$. Given a section $s$ of $A$ and a point $y \in \operatorname{graph}(s)$, we have a splitting $T_{y} A=$ $V_{y} \oplus T_{y} \operatorname{graph}(s)$ of the tangent space to $A$ at $y$, where $V:=\operatorname{ker}(d \pi)$ is the vertical bundle. We will denote by $\mathrm{p}_{s}^{\mathrm{v}}$ the projection $T_{y} A \rightarrow V_{y}$ with kernel $T_{y} \operatorname{graph}(s)$.

\footnotetext{
${ }^{5}$ [16. Prop. 1.15] is stated for bivector fields, but it carries over immediately to the case of vector fields.
} 
Lemma 3.13. Let $X_{t}$ be a one-parameter family of vector fields on $A$, and $\phi_{t}$ its flow. Moreover, let $s_{t}$ be a one-parameter family of sections of $A$ such that

$$
\operatorname{graph}\left(s_{t}\right)=\phi_{t}\left(\operatorname{graph}\left(s_{0}\right)\right)
$$

holds for all $t \in[0,1]$.

Then $s_{t}$ satisfies the equation

$$
\frac{\partial}{\partial t} s_{t}=\mathrm{p}_{s_{t}}^{\mathrm{v}} X_{t}, \quad \forall t \in[0,1],
$$

which we see as an equality of sections of $\left.V\right|_{\operatorname{graph}\left(s_{t}\right)}$.

Proof. If we define $\psi_{t}$ to be the isotopy of $M$ given by $\pi \circ \phi_{t} \circ s_{0}$, we have

$$
s_{t}=\phi_{t} \circ s_{0} \circ\left(\psi_{t}\right)^{-1}: M \rightarrow A .
$$

Evaluating at $x \in M$ and taking the time derivative we obtain

$$
\frac{\partial}{\partial t}\left(s_{t}(x)\right)=\left.X_{t}\right|_{s_{t}(x)}+\left(\phi_{t}\right)_{*}\left(s_{0}\right)_{*} \frac{\partial}{\partial t}\left(\left(\psi_{t}\right)^{-1}(x)\right) .
$$

We finish noticing that the last summand is tangent to $\phi_{t}\left(\operatorname{graph}\left(s_{0}\right)\right)=\operatorname{graph}\left(s_{t}\right)$, and that $\frac{\partial}{\partial t}\left(s_{t}(x)\right)$ lies in $V_{s t}(x)$.

The following Lemma, whose (geometric) proof was communicated to us by Luca Vitagliano, is a converse to Lemma 3.13 .

Lemma 3.14. Let $X_{t}$ be a one-parameter family of vector fields on $A$, and $\phi_{t}$ its flow, assumed to exist for all $t \in[0,1]$. Suppose $s_{t}$ is a one-parameter family of sections of $A$ that satisfies

$$
\frac{\partial}{\partial t} s_{t}=\mathrm{p}_{s_{t}}^{\mathrm{v}} X_{t}, \quad \forall t \in[0,1] .
$$

Then the family of submanifolds graph $\left(s_{t}\right)$ coincides with $\phi_{t}\left(\operatorname{graph}\left(s_{0}\right)\right)$ for all $t \in[0,1]$.

Proof. We work on the vector bundle $A \times[0,1] \rightarrow M \times[0,1]$, and denote by $t$ the standard coordinate on the $[0,1]$-factor. Define $\widehat{s} \in \Gamma(A \times[0,1])$ by

$$
\widehat{s}(x, t)=\left(s_{t}(x), t\right)
$$

and the vector field $\widehat{X}$ on $A \times[0,1]$ by

$$
\left.\widehat{X}\right|_{(y, t)}=\left.\left(X_{t}\right)\right|_{y}+\frac{\partial}{\partial t} .
$$

Notice that the flow $\varphi_{t}$ of $\widehat{X}$ takes $(y, 0)$ to $\left(\phi_{t}(y), t\right)$ for all $y \in A$.

The key observation is that the vector field $\widehat{X}$ is tangent to the submanifold $\operatorname{graph}(\widehat{s})$. To this end we compute

$$
\frac{d}{d t} \widehat{s}(x, t)=\frac{d}{d t} s_{t}(x)+\frac{\partial}{\partial t}=\left.\left(X_{t}\right)\right|_{s_{t}(x)}-v+\frac{\partial}{\partial t}=\left.\widehat{X}\right|_{\left(s_{t}(x), t\right)}-v
$$

for some vector $v \in T_{s_{t}(x)}\left(\operatorname{graph}\left(s_{t}\right)\right)$, making use of equation (4) in the second equality. This implies that $\left.\widehat{X}\right|_{\left(s_{t}(x), t\right)}=\frac{d}{d t} \widehat{s}(x, t)+v$ is the sum of two vectors tangent to graph $(\widehat{s})$. 
Hence the flow $\varphi_{t}$ of $\widehat{X} \operatorname{maps} \operatorname{graph}\left(\left.\widehat{s}\right|_{M \times\{0\}}\right)=\operatorname{graph}\left(s_{0}\right) \times\{0\}$ to $\operatorname{graph}\left(\left.\widehat{s}\right|_{M \times\{t\}}\right)=$ $\operatorname{graph}\left(s_{t}\right) \times\{t\}$. On the other hand, we saw above that $\varphi_{t} \operatorname{maps} \operatorname{graph}\left(s_{0}\right) \times\{0\}$ to $\phi_{t}\left(\operatorname{graph}\left(s_{0}\right)\right) \times\{t\}$.

In Lemma 3.14 we assume that the flow of $X_{t}$ is defined on the interval $[0,1]$. We now show that this assumption can be replaced by asking that the base $M$ of the vector bundle be compact.

Lemma 3.15. Let $\pi: A \rightarrow M$ be a vector bundle over a compact base $M$. Let $X_{t}$ be a one-parameter family of vector fields on $A$ and $s_{t}$ a one-parameter family of sections of $A$ that satisfies

$$
\frac{\partial}{\partial t} s_{t}=\mathrm{p}_{s_{t}}^{\mathrm{v}} X_{t}, \quad \forall t \in[0,1]
$$

Then the flow lines of $X_{t}$ starting at graph $\left(s_{0}\right)$ exist for $t \in[0,1]$ and the equality

$$
\operatorname{graph}\left(s_{t}\right)=\phi_{t}\left(\operatorname{graph}\left(s_{0}\right)\right)
$$

holds.

Proof. Fix an auxiliary fibre metric on $A$. We let $K \subset A$ be the compact subset given by all vectors of length less than or equal to $l+\delta$ for some $\delta>0$, where

$$
l:=\max _{x \in M, t \in[0,1]}\left(\left\|s_{t}(x)\right\|\right) .
$$

Let $\varphi$ be a function on $A$ with compact support, and so that $\left.\varphi\right|_{K} \equiv 1$. Then $\left(\varphi X_{t}\right)_{t \in[0,1]}$ is a time-dependent vector field whose integral curves are defined for all times. Let $T$ be the maximal element of $[0,1]$ such that $\operatorname{graph}\left(s_{t}\right)=\phi_{t}\left(\operatorname{graph}\left(s_{0}\right)\right)$ holds for all $t \in[0, T]$. Suppose $T<1$. There is $\epsilon>0$ such that $\phi_{t}\left(\operatorname{graph}\left(s_{0}\right)\right) \subset K$ for all $t \in[0, T+\epsilon]$. But since the one-parameter families $X_{t}$ and $\varphi X_{t}$ agree on $K$, we see as in Lemma 3.14 that $\operatorname{graph}\left(s_{t}\right)=\phi_{t}\left(\operatorname{graph}\left(s_{0}\right)\right)$ actually holds for all $t \in[0, \min \{1, T+\epsilon\}]$, which is a contradiction.

Remark 3.16. The compactness assumption in Lemma 3.15 can not be omitted, as the following counter-example shows. Take a non-compact manifold $M$, a vector field $X$ on $M$ whose flow is not defined on the whole of $[0,1]$. Take the trivial bundle $A:=M \times[0,1]$ and let $X_{t}$ be the horizontal lift of $X$ to $A$. Moreover, let $\operatorname{graph}\left(s_{t}\right)$ be $M \times\{0\}$. Notice that $\frac{\partial}{\partial t} s_{t}$ and $\mathrm{p}_{s_{t}}^{\mathrm{v}} X_{t}$ agree, since they both vanish identically.

3.5. Hamiltonian equivalence $=$ gauge-equivalence. Our aim is to compare the two equivalence relations $\sim_{\text {gauge }}$ and $\sim_{\text {Ham }}$ on $\operatorname{Def}_{U}(E)$. As an intermediate notion we introduce:

Definition 3.17. One can restrict Hamiltonian equivalence $\sim_{\mathrm{Ham}}$ by only allowing Hamiltonian flows generated by functions of the type $\pi^{*} f$, with $f \in \mathcal{C}^{\infty}(C)$. We call the resulting equivalence relation base Hamiltonian equivalence and denote it by $\sim_{b \mathrm{Ham}}$. 
Proposition 3.18. The following chain of implications holds between the three equivalence relations on $\operatorname{Def}_{U}(C)$ :

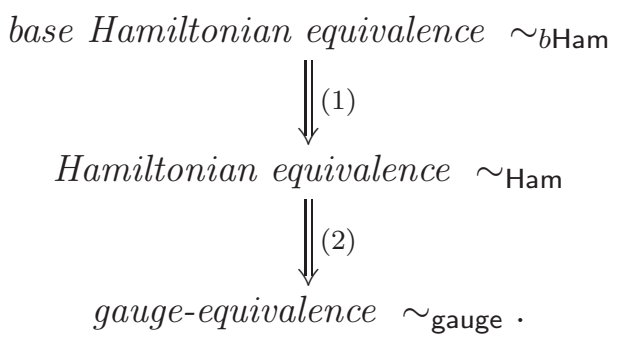

Proof. Implication (1) is clear, so we pass on to implication (2). Let $s_{t}$ be a smooth family of coisotropic sections of $U$ and suppose that $H_{t}$ is a smooth family of functions on $U$ such that the Hamiltonian flow $\phi_{t}^{H_{t}}$ of $H_{t}$ maps $\operatorname{graph}\left(s_{0}\right)$ to $\operatorname{graph}\left(s_{t}\right)$. By Lemma 3.13, this implies that the equation

$$
\frac{\partial}{\partial t} s_{t}=\mathrm{p}_{s_{t}}^{\mathrm{v}} X_{H_{t}}
$$

holds for all $t \in[0,1]$. Define $f_{t} \in \mathcal{C}^{\infty}(C)$ to be $H_{t} \circ s_{t}$.

Observe that $\mathrm{p}_{s_{t}}^{\mathrm{v}}\left(X_{H_{t}}-X_{\pi^{*} f_{t}}\right)$ is zero since $H_{t}-\pi^{*} f_{t}$ vanishes on $\operatorname{graph}\left(s_{t}\right)$ and consequently $X_{H_{t}-\pi^{*} f_{t}}=X_{H_{t}}-X_{\pi^{*} f_{f}}$ gets mapped to $T \operatorname{graph}\left(s_{t}\right)$ under $\Pi^{\sharp}$, $\operatorname{since} \operatorname{graph}\left(s_{t}\right)$ is coisotropic. We conclude that the equation

$$
\frac{\partial}{\partial t} s_{t}=\mathrm{p}_{s_{t}}^{\mathrm{v}} X_{H_{t}}=\mathrm{p}_{s_{t}}^{\mathrm{v}}\left(X_{\pi^{*} f_{t}}\right)
$$

holds. By Proposition 3.11 we have that $s_{0}$ and $s_{1}$ are gauge-equivalent as claimed.

Under the assumption that $C$ is compact, we can "close the circle" of the implications of Proposition 3.18.

Proposition 3.19. Suppose $C$ is compact coisotropic submanifold. Then the following implication holds for the local symplectic model of $C$ :

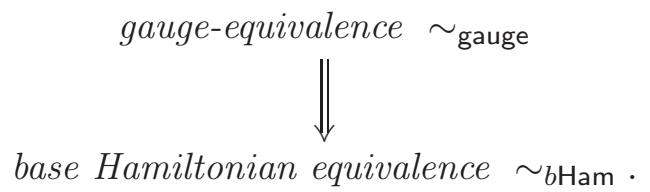

Proof. Suppose that $s_{0}$ and $s_{1}$ of $\operatorname{Def}_{U}(C)$ are gauge-equivalent. This means that there is a one-parameter family $s_{t}$ in $\operatorname{Def}_{U}(C)$ and a one-parameter family of functions $f_{t}$ on $C$ such that

$$
\frac{\partial}{\partial t} s_{t}=\mathrm{p}_{s_{t}}^{\mathrm{v}} X_{\pi^{*}} f_{t}
$$

holds for all $t \in[0,1]$.

The compactness of $C$ allows us to apply Lemma 3.15, which states that the flow $\phi_{t}$ of $X_{\pi^{*} f_{t}}$ exists for all $t \in[0,1]$ and indeed maps $\operatorname{graph}\left(s_{0}\right)$ to $\operatorname{graph}\left(s_{t}\right)$. 
Remark 3.20. When $C$ is a Lagrangian submanifold, Hamiltonian equivalence implies base Hamiltonian equivalence without any compactness assumption: this follows from Proposition 3.18 and Proposition 3.19, noticing that in the latter in the Lagrangian case no compactness is necessary, for $X_{\pi^{*} f_{t}}$ is a vertical vector field on $U \subset T^{*} C$. In particular, if $\left(\phi_{t}\right)_{t \in[0,1]}$ is an isotopy by Hamiltonian diffeomorphisms mapping the zero section $C$ to sections of $U$ for all $t \in[0,1]$, then $\phi_{1}(C)$ is the graph of an exact 1-form on $C$. This is in agreement with [11, Proposition 9.33].

Combining Proposition 3.18 and Proposition 3.19 we arrive at the main result of this section:

Theorem 3.21. Let $C$ be a compact coisotropic submanifold with local symplectic model $(U, \Omega)$. The equivalence relations on

$$
\operatorname{Def}_{U}(C):=\{s \in \Gamma(U): s \text { is coisotropic }\}
$$

given by

- Hamiltonian equivalence $\sim_{\text {Ham }}$ (Definition 3.1) and

- gauge-equivalence $\sim_{\text {gauge }}$ (Definition [3.9, see also Proposition 3.11)

coincide.

As a consequence we obtain the following result:

Theorem 3.22. Let $C$ be a compact coisotropic submanifold with local symplectic model $(U, \Omega)$. The bijection

$$
\operatorname{Def}_{U}(C) \cong \mathrm{MC}_{U}(\Gamma(\wedge E)[1])
$$

descends to a bijection

$$
\mathcal{M}_{U}^{\mathrm{Ham}}(C):=\operatorname{Def}_{U}(C) / \sim_{\mathrm{Ham}} \cong \mathrm{MC}_{U}(\Gamma(\wedge E)[1]) / \sim_{\text {gauge }} .
$$

Remark 3.23.

(1) One could use Theorem 3.22 to rederive the infinitesimal description of $\mathcal{M}_{U}^{\mathrm{Ham}}(C)$ from Subsection 3.2 by linearizing the Maurer-Cartan equation and the gaugeequivalence.

(2) A description of $\mathcal{M}_{U}^{\mathrm{Ham}}(C)$ similar to Theorem 3.22 was obtained in [15. There the differential graded Lie algebra associated to the BFV-complex was used to encode deformations of $C$ and the action of Hamiltonian diffeomorphisms. The BFVcomplex has the advantage that it works for arbitrary Poisson structures, unlike the $L_{\infty}$ [1]-algebra from [12] and [2]. The drawbacks of the approach relying on the BFVcomplex is that one needs to single out the geometrically relevant Maurer-Cartan elements by hand and is forced to deal with symmetries of symmetries.

\section{Symplectomorphisms}

Next we consider the action of symplectomorphisms on the space of coisotropic sections, which we encode by an equivalence relation $\sim$ Sym on the space of coisotropic sections $\operatorname{Def}_{U}(C)$. In the search for an interpretation of $\sim$ Sym in terms of Oh and Park's $L_{\infty}[1]-$ algebra, we are led to reconsider Voronov's derived bracket construction [18, 19]. 
4.1. The deformation problem. Let $C$ be a coisotropic submanifold with local symplectic model $(U, \Omega)$.

Definition 4.1. Two coisotropic sections $s_{0}$ and $s_{1}$ of $U$ are called symplectic equivalent, $s_{0} \sim \sim_{\text {Sym }} s_{1}$ if there is a family of coisotropic sections $s_{t} \in \Gamma(U)$, agreeing with the given ones at $t=0$ and $t=1$, and an isotopy of local symplectomorphisms $\phi_{t}$ such that $\phi_{t}$ maps $\operatorname{graph}\left(s_{0}\right)$ to $\operatorname{graph}\left(s_{t}\right)$ for all $t \in[0,1]$.

Remark 4.2. As for Hamiltonian equivalence, it is straight-forward to check that $\sim_{\text {Sym }}$ is in fact an equivalence relation. We define the symplectic moduli space of coisotropic sections to be the set

$$
\mathcal{M}_{U}^{\text {Sym }}(C):=\operatorname{Def}_{U}(C) / \sim_{\text {Sym }}
$$

Our aim is to answer

$$
\text { How can one describe the set } \mathcal{M}_{U}^{\text {Sym }}(C) \text { ? }
$$

which we will achieve in Theorem 4.19 of Subsection 4.4.

4.2. Infinitesimal moduli. We first consider the infinitesimal counterpart of $\mathcal{M}_{U}^{\text {Sym }}(C)$. We argue - see Remark 4.6 - that the formal tangent space to $\mathcal{M}_{U}^{\mathrm{Sym}}(C)$ at the equivalence class of the zero-section $C$ is given by the cokernel of a certain map $r: H^{1}(C) \rightarrow H_{\mathfrak{F}}^{1}(C)$.

\section{Remark 4.3.}

(1) Recall that every coisotropic submanifold $C$ comes equipped with a pre-symplectic structure $\omega_{C}$, whose kernel $K$ is an involutive distribution. The corresponding foliation of $C$ is denoted by $\mathfrak{F}$. Restriction to $K$ yields a chain map

$$
r: \Omega(C) \rightarrow \Omega_{\mathfrak{F}}(C)
$$

between the ordinary and the foliated de Rham complex of $C$.

(2) As we observed in Subsection 2.2, $\Omega_{\mathfrak{F}}(C)$ is isomorphic to $\Gamma(\wedge E)$, equipped with the differential $P([\Pi, \cdot])$, where $P$ is the projection from multivector-fields on $E$ onto $\Gamma(\wedge E)$.

Lemma 4.4. Let $C$ be a coisotropic submanifold of $(E, \omega)$ with inclusion map $\iota$. Given $\beta \in \Omega^{1}(E)$, denote by $X_{\beta}$ the unique vector field on $E$ which satisfies

$$
i_{X_{\beta}} \omega=\beta \text {. }
$$

Then the triangle

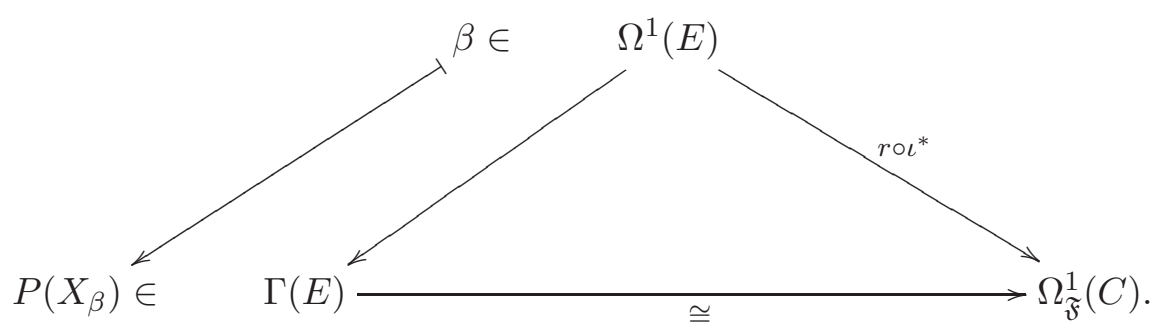

commutes. 
Proof. The identification $E \cong K^{*}$ from Section 1 which is used in the bottom map of the above diagram, maps $e \in E_{x}$ to $\left.\omega^{\sharp}(e)\right|_{K_{x}}$. We have

$$
\left.\omega^{\sharp}\left(P\left(X_{\beta}\right)\right)\right|_{K}=\left.\omega^{\sharp}\left(X_{\beta}\right)\right|_{K}=\left.\beta\right|_{K},
$$

where in the first equality we used that $\omega(v,-)$ vanishes on $K$ for all $v \in T C$. This proves the desired commutativity.

The following proposition is a special instance of Lemma 6.7 in [9, Subsection 6.3.], where the more general case of locally conformal symplectic manifolds is treated. In its formulation we make use of the above isomorphism in order to view $\left.\frac{\partial s_{t}}{\partial t}\right|_{t=0} \in \Gamma(E)$ as an element of $\Omega_{\mathfrak{F}}^{1}(C)$.

Proposition 4.5. Suppose that $\left(s_{t}\right)_{t \in[0,1]}$ is a family of coisotropic sections that starts at the zero-section and is trivial under symplectic equivalence, i.e. there is a symplectic isotopy $\phi_{t}$ such that the image of the zero section under $\phi_{t}$ coincides with the graph of $s_{t}$.

Then the cohomology class of $\left.\frac{\partial s_{t}}{\partial t}\right|_{t=0}$ in $H_{\mathfrak{F}}^{1}(C)$ lies in the image of $r: H^{1}(C) \rightarrow H_{\mathfrak{F}}^{1}(C)$.

Proof. Suppose that $\phi_{t}$ is the symplectic isotopy generated by the family of vector fields $X_{t}$. Since $\phi_{t}$ is symplectic, $\beta_{t}:=i_{X_{t}} \omega$ is a family of closed one-forms. By Lemma 3.13, we can write $\left.\frac{\partial s_{t}}{\partial t}\right|_{t=0}$ as $P\left(X_{0}\right)$. By the previous lemma, this equals the image of $\beta_{0}$ under $r \circ \iota^{*}$. In particular, the cohomology class of $\left.\frac{\partial s_{t}}{\partial t}\right|_{t=0}$ coincides with the cohomology class $\left(r \circ \iota^{*}\right)\left[\beta_{0}\right]$, hence lies in the image of $r: H^{1}(C) \rightarrow H_{\mathfrak{F}}^{1}(C)$.

Remark 4.6. Proposition 4.5 is an analogue of Proposition 3.5, where we showed that if a family $\left(s_{t}\right)_{t \in[0,1]}$ is trivial under Hamiltonian equivalence then the cohomology class of $\left.\frac{\partial s_{t}}{\partial t}\right|_{t=0}$ is zero.

One can strengthen Proposition 4.5 by observing that, by the same proof, every element in the image of the map $r: \Omega_{\text {closed }}^{1}(C) \rightarrow \Omega_{\mathfrak{F} \text {,closed }}^{1}(C)$ is of the form $\left.\frac{\partial s_{t}}{\partial t}\right|_{t=0}$, where $\left(s_{t}\right)_{t \in[0, \epsilon)}$ arises through the action of a symplectic isotopy on the zero-section. Indeed, for every $\gamma \in$ $\Omega_{\text {closed }}^{1}(C)$ one considers the symplectic isotopy generated by the vector field $\left(\omega^{\sharp}\right)^{-1}\left(\pi^{*} \gamma\right)$.

In full analogy to Remark 3.6, this together with Remark 2.6 shows that the formal tangent space at zero to $\mathcal{M}_{U}^{\mathrm{Sym}}(C)$ is

$$
\Omega_{\mathfrak{F} \text {,closed }}^{1} / r\left(\Omega_{\text {closed }}^{1}(C)\right) \cong H_{\mathfrak{F}}^{1}(C) / r\left(H^{1}(C)\right),
$$

that is, the cokernel of $r: H^{1}(C) \rightarrow H_{\mathfrak{F}}^{1}(C)$. The isomorphism is obtained by quotienting both terms on the left-hand side by $\Omega_{\mathfrak{F} \text {,exact }}^{1}$ and by using the following linear algebra statement for the denominator: if $f: V_{1} \rightarrow V_{2}$ is a linear map and $W_{1}, W_{2}$ are subspaces such that $f\left(W_{1}\right)=W_{2}$, then $f\left(V_{1}\right) / W_{2}=\operatorname{Im}\left([f]: V_{1} / W_{1} \rightarrow V_{2} / W_{2}\right)$.

We note that if $C$ is Lagrangian we have $H_{\mathfrak{F}}^{1}(C)=H^{1}(C)$ and $r$ is the identity, so its cokernel is trivial, as expected.

Notice also, by the above and Remark 3.6, that the formal tangent space at zero of $\mathcal{M}_{U}^{\text {Sym }}(C)$ is a quotient of the formal tangent space to $\mathcal{M}_{U}^{\text {Ham }}(C)$, and that they agree iff $r: H^{1}(C) \rightarrow H_{\mathfrak{F}}^{1}(C)$ is the zero map. This happens for instance if $H^{1}(C)=0$, in which cases it is clear a priori that $\mathcal{M}_{U}^{\text {Sym }}(C)=\mathcal{M}_{U}^{\text {Ham }}(C)$, for all symplectic vector fields on $U$ are Hamiltonian. In Example 4.24 below we display an example in which $r$ is not the zero map. 
4.3. The extended formal picture. We explain now how to interpret the equivalence relation $\sim$ Sym from the point of view of Oh and Park's $L_{\infty}[1]$-algebra structure on $\Gamma(\wedge E)[1]$.

To this aim, we first need to briefly recall Voronov's derived bracket construction [18, 19].

Remark 4.7 (on Voronov's derived brackets).

(1) Let $L$ be a graded Lie algebra, $\mathfrak{a}$ an abelian subalgebra and $P: L \rightarrow \mathfrak{a}$ a projection whose kernel is a Lie subalgebra. Furthermore, suppose $X$ is a Maurer-Cartan element of $L$, i.e. $X \in L_{1}$ satisfying $[X, X]=0$, such that $P(X)=0$. In [18], Voronov showed that the derived brackets

$$
\lambda_{k}\left(a_{1} \otimes \cdots \otimes a_{k}\right):=P\left(\left[\cdots\left[\left[X, a_{1}\right], a_{1}\right] \cdots, a_{k}\right]\right)
$$

equip $\mathfrak{a}$ with the structure of an $L_{\infty}[1]$-algebra.

(2) Observe that $X$ gives rise to a coboundary operator $-[X, \cdot]$ on $L$, which makes $L$ into a differential graded Lie algebra. This DGLA structure on $L$, the $L_{\infty}$ [1]-algebra structure on $\mathfrak{a}$ described above, and additional structure maps $\lambda_{i}(i \geq 1)$ combine into an $L_{\infty}[1]$-algebra structure on $L[1] \oplus \mathfrak{a}$, see [18, 19]. The additional structure maps take values in $\mathfrak{a}$ and are given by

$$
\lambda_{k+1}\left(l[1] \otimes a_{1} \otimes \cdots \otimes a_{k}\right):=P\left(\left[\cdots\left[\left[l, a_{1}\right], a_{2}\right] \cdots, a_{k}\right]\right),
$$

where $l \in L$ and $k \geq 0, a_{1}, \cdots, a_{k} \in \mathfrak{a}$. Notice that for $k=0$ we obtain $\lambda_{1}(l[1])=$ $P(l)$.

Since $\mathfrak{a}$ is a $L_{\infty}[1]$-subalgebra of $L[1] \oplus \mathfrak{a}$, the inclusion $\beta \mapsto(0, \beta)$ identifies MaurerCartan elements of $\mathfrak{a}$ with those Maurer-Cartan elements of $L[1] \oplus \mathfrak{a}$ which lie in $\{0\} \oplus \mathfrak{a}$. We use this identification to obtain a new equivalence relation on $\operatorname{MC}(\mathfrak{a})$. To this aim, we need to modify $L[1] \oplus \mathfrak{a}$ slighty to guarantee that the set of Maurer-Cartan elements in $\{0\} \oplus \mathfrak{a}$ is preserved by the gauge-action:

Lemma 4.8. Let $Z(X) \subset L$ be the graded Lie subalgebra of elements $\sigma$ which commute with $X$.

(1) $Z(X)[1] \oplus \mathfrak{a} \subset L[1] \oplus \mathfrak{a}$ is an $L_{\infty}[1]$-subalgebra.

(2) The gauge-equivalence in $Z(X)[1] \oplus \mathfrak{a}$ preserves the set of Maurer-Cartan elements in $\{0\} \oplus \mathfrak{a} \subset L[1] \oplus \mathfrak{a}$.

Proof. The first claim reduces to the fact that $Z(X)$ is a graded Lie subalgebra of $L$.

Concerning the second claim, we consider the effect of the gauge-action on first component of $L[1] \oplus \mathfrak{a}$. We find

$$
\frac{d}{d t} l_{t}=\left[X, \sigma_{t}\right]+\left[l_{t}, \sigma_{t}\right]
$$

where $\sigma_{t}$ is a family of elements in $L_{0}$ and $l_{t}$ in $L_{1}$. Now if we require $\sigma_{t}$ to lie in $Z(X)$, the term $\left[X, \sigma_{t}\right]$ is zero and we recover the usual adjoint action of $L_{0}$ on $L_{1}$, for which the origin is clearly a fixed point.

Remark 4.9. The restriction to Maurer-Cartan elements in $\{0\} \oplus \mathfrak{a}$ of the gauge-equivalence of $Z(X)[1] \oplus \mathfrak{a}$ can be alternatively described as follows: It is straight-forward to check that if $L^{\prime}$ is any graded Lie subalgebra of $L$ closed under $[X, \cdot]$, then $L^{\prime}[1] \oplus \mathfrak{a}$ is closed w.r.t. all the multibrackets of the $L_{\infty}[1]$-algebra $L[1] \oplus \mathfrak{a}$. We apply this to $L^{\prime}=Z_{0}(X)$, the degree zero component of $Z(X)$, to obtain an $L_{\infty}[1]$-algebra $Z_{0}(X)[1] \oplus \mathfrak{a}$. Notice that 
$\mathrm{MC}\left(Z_{0}(X)[1] \oplus \mathfrak{a}\right)=\mathrm{MC}(\{0\} \oplus \mathfrak{a})$, simply because $Z_{0}(X)[1]$ is concentrated in degree -1 while Maurer-Cartan elements have degree zero. Hence the gauge-equivalence of $Z_{0}(X)[1] \oplus \mathfrak{a}$ on its Maurer-Cartan elements agrees with the the restriction of the gauge-equivalence appearing in Lemma 4.8 .

This result prompts us to give the following definition

Definition 4.10. Two Maurer-Cartan elements $\beta_{0}$ and $\beta_{1}$ of $\mathfrak{a}$ are called extended gaugeequivalent, written $\beta_{0} \sim_{\text {ext-gauge }} \beta_{1}$, if there is a one-parameter family $\sigma_{t}$ of degree 0 elements of $L$ which commute with $X$ and a one-parameter family $\beta_{t}$ of elements of $\mathfrak{a}_{0}$, agreeing with the given ones at $t=0$ and $t=1$, such that

$$
\frac{\partial}{\partial t} \beta_{t}=P\left(\sigma_{t}\right)+P\left(\left[\sigma_{t}, \beta_{t}\right]\right)+\frac{1}{2 !} P\left(\left[\left[\sigma_{t}, \beta_{t}\right], \beta_{t}\right]\right)+\frac{1}{3 !} P\left(\left[\left[\left[\sigma_{t}, \beta_{t}\right], \beta_{t}\right], \beta_{t}\right]\right)+\ldots
$$

holds for all $t \in[0,1]$.

We note that in the above definition we only allow gauge-equivalences generated by elements coming from the component $L[1]$, which seems more restrictive than considering arbitrary gauge-equivalences in $Z(X)[1] \oplus \mathfrak{a}$. However, observe that families of elements of the form $\left[X, \gamma_{t}\right]$, for $\gamma_{t} \in \mathfrak{a}_{-1}$, automatically commute with $X$ and hence give rise to extended gauge-equivalences. If we substitute such a family $\left[X, \gamma_{t}\right]$ for $\sigma_{t}$ in the above formula, we obtain

$\frac{\partial}{\partial t} \beta_{t}=P\left(\left[X, \gamma_{t}\right]\right)+P\left(\left[\left[X, \gamma_{t}\right], \beta_{t}\right]\right)+\frac{1}{2 !} P\left(\left[\left[\left[X, \gamma_{t}\right], \beta_{t}\right], \beta_{t}\right]\right)+\frac{1}{3 !} P\left(\left[\left[\left[\left[X, \gamma_{t}\right], \beta_{t}\right], \beta_{t}\right], \beta_{t}\right]\right)+\ldots$

This expression coincides with the defining formula of an (ordinary) gauge-equivalence between the Maurer-Cartan elements $\beta_{0}$ and $\beta_{1}$, see Definition 3.8 in Subsection 3.3. Hence $\sim_{\text {ext-gauge }}$ from Definition 4.10 really coincides with the gauge-equivalence inherited from $Z(X)[1] \oplus \mathfrak{a}$ and we furthermore see that ordinary gauge-equivalence implies extended gaugeequivalence.

Remark 4.11. One can obtain every $L_{\infty}[1]$-algebra from the derived bracket construction, see [18, Example 4.1] and [4, Appendix A.3] for details: Let $W$ be a graded vector space and denote its graded symmetric coalgebra by $S W:=\oplus_{i>0} S^{i} W$, where $S^{i} W$ can be described as the fixed point set of the $i$-fold tensor algebra $T^{i} W$ on $W$ under the even action of the symmetric group $\Sigma_{i}$. The deconcatenation map $\Delta: T W \rightarrow T W \otimes T W$ given by

$$
\begin{aligned}
\Delta\left(x_{1} \otimes \cdots \otimes x_{n}\right):= & 1 \otimes\left(x_{1} \otimes \cdots \otimes x_{n}\right)+\sum_{i=1}^{n-1}\left(x_{1} \otimes \cdots \otimes x_{i}\right) \otimes\left(x_{i+1} \otimes \cdots \otimes x_{n}\right)+ \\
& \left(x_{1} \otimes \cdots \otimes x_{n}\right) \otimes 1
\end{aligned}
$$

restricts to $S W$ and defines a cocommutative coassociative coproduct there. As essentially observed by Stasheff in [17], an $L_{\infty}$ [1]-algebra structure on $W$ is the same as a degree 1 coderivation $D$ of the coalgebra $S W$ that annihilates $1 \in \mathbb{R} \subset S W$ and squares to zero, i.e. an endomorphism $D$ of $S W$ that satisfies

$$
\Delta \circ D=(D \otimes \mathrm{id}+\mathrm{id} \otimes D) \circ \Delta, \quad D(1)=0, \quad \text { and } \quad D \circ D=0 .
$$


This means that an $L_{\infty}[1]$-algebra structure on $W$ corresponds to a Maurer-Cartan element $D$ in the graded Lie algebra of coderivations $\operatorname{Coder}(S W)$, equipped with the commutator bracket.

One can reinterpret this construction in terms of the higher derived bracket construction as follows: For $L$ we take $\operatorname{Coder}(S W)$ and as the abelian subalgebra we take $W$, which sits inside $\operatorname{Coder}(S W) \cong \operatorname{Hom}(S W, W)$ as those homomorphisms which map 1 to an element of $W$ and everything else to 0 . The projection map $P: \operatorname{Coder}(S W) \cong \operatorname{Hom}(S W, W) \rightarrow W$ is evaluation at $1 \in \mathbb{R}=S^{0} W$ and the Maurer-Cartan element $X$ is the coderivation $D$. The corresponding derived brackets just return the $L_{\infty}[1]$-algebra structure on $W$.

To see what extended gauge-equivalence means in this case, let $\sigma_{t} \in \operatorname{Coder}(S W)$ be a family of coderivation of degree 0 which commutes with $D$. The extended gauge-action on Maurer-Cartan elements $\beta_{t}$ of $W$ reads

$$
\frac{d}{d t} \beta_{t}=\operatorname{pr}_{W}\left(\sigma_{t}+\sigma_{t}\left(\beta_{t}\right)+\frac{1}{2} \sigma_{t}\left(\beta_{t} \otimes \beta_{t}\right)+\cdots\right),
$$

where $\operatorname{pr}_{W}$ denotes the projection $S W \rightarrow W$.

Suppose we can integrate this family of coderivations to a family of automorphisms $\Phi_{t}$ of the coalgebra $S W$. By construction, $\Phi_{t}$ will commute with $D$ as well and act on MaurerCartan elements of $W$ by

$$
\operatorname{pr}_{W}\left(\Phi_{t}\left(1+\beta+\frac{1}{2} \beta \otimes \beta+\frac{1}{3 !} \beta \otimes \beta \otimes \beta+\cdots\right)\right) .
$$

This formula can by verified by checking that differentiation yields the formula for the extended gauge-action from above.

In short, extended gauge-equivalence in the case at hand amounts to the action of those automorphisms of the $L_{\infty}[1]$-algebra structure $D$ which are connected to the identity.

We now return to the equivalence relation $\sim_{\text {Sym }}$ on the space of coisotropic deformations. If one applies Voronov's derived bracket construction (see Remark 4.7) to the data

- $L=\left(\chi^{\bullet}(E)[1],[-,-]\right)$,

- $\mathfrak{a}=\Gamma(\wedge E)[1]$

- $P: L \rightarrow \mathfrak{a}$ the projection as before,

- $X=\Pi \in \chi^{2}(E)$ the Poisson bivector field corresponding to $\omega$,

one recovers Oh and Park's $L_{\infty}[1]$-algebra structure on $\Gamma(\wedge E)[1]$ from Subsection 2.3 .

By Lemma 4.8, its Maurer-Cartan elements are endowed with a second equivalence relation, arising from the degree 0-elements of $\chi^{\bullet}(E)[1]$ that commute with the Poisson bivector field. These are exactly the symplectic vector fields. Lemma 4.8 prompts us to repeat the definition of gauge-equivalence from Subsection 3.3. with the Hamiltonian vector fields $X_{\pi^{*}} f_{t}$ replaced with any family of symplectic vector fields. However, in order to maintain the link to geometry, we restrict ourselves to symplectic vector fields on $E$ which are firbre-wise entrie.

Definition 4.12. Let $(U, \Omega)$ be a local symplectic model for the coisotropic submanifold $C$.

Two elements $s_{0}$ and $s_{1}$ of $\operatorname{Def}_{U}(C)$ are extended gauge-equivalent, $s_{0} \sim$ ext-gauge $s_{1}$, if there is a one-parameter family $s_{t} \in \Gamma(U)$, agreeing with $s_{0}$ and $s_{1}$ at the endpoints, and 
a family of symplectic, firbre-wise entrie vector fields $X_{t}$ on $U$ such that

$$
\frac{\partial}{\partial t}\left(-s_{t}\right)=P\left(e^{\left[\cdot,-s_{t}\right]} X_{t}\right)
$$

holds for all $t \in[0,1]$.

Remark 4.13. We denote the induced equivalence relation on $\operatorname{Def}_{U}(C)$ by $\sim_{\text {ext-gauge. The }}$ proof of Proposition 3.11 goes through mutatis mutandis and we obtain:

Proposition 4.14. Elements $s_{0}$ and $s_{1}$ of $\operatorname{Def}_{U}(C)$ are extended gauge-equivalent if and only if there is a one-parameter family $s_{t} \in \Gamma(U)$, agreeing with $s_{0}$ and $s_{1}$ at the endpoints, and a one-parameter family $X_{t}$ of symplectic and firbre-wise entrie vector fields on $U$ such that

holds for all $t \in[0,1]$.

$$
\frac{\partial}{\partial t} s_{t}=\mathrm{p}_{s_{t}}^{\mathrm{v}}\left(\left.X_{t}\right|_{\operatorname{graph}\left(s_{t}\right)}\right)
$$

4.4. Symplectic equivalence $=$ extended gauge-equivalence. Our aim is to compare the two equivalence relations $\sim_{\text {ext-gauge }}$ and $\sim_{\text {Sym }}$ on $\operatorname{Def}_{U}(E)$.

Remark 4.15. The following two results are proved in parallel to Proposition 3.18 and Proposition 3.19. The key point is the following: if we are given a section $s$ of $U$ whose graph is coisotropic, and a closed 1-form $\beta$ on $E$, the vector fields $\left(\omega^{\sharp}\right)^{-1}\left(\pi^{*} s^{*} \beta\right)$ and $\left(\omega^{\sharp}\right)^{-1}(\beta)$ have the same vertical projection onto $\left.E\right|_{\operatorname{graph}(s)}$ along $T \operatorname{graph}(s)$. As in the proofs of Proposition 3.18 and Propositions 3.19, this fact allows one to replace any family of symplectic isotopies by a family of symplectic isotopies generated by firbre-wise entrie symplectic vector fields.

Proposition 4.16. The following implication holds between the equivalence relations on $\operatorname{Def}_{U}(C)$ :

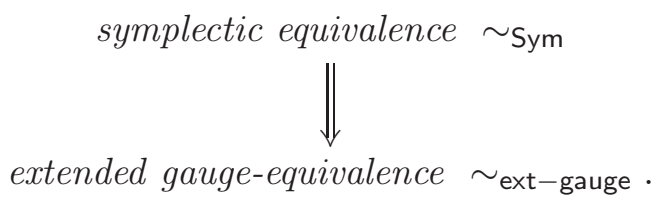

Under the assumption that $C$ is compact, we can reverse the implications of Proposition 4.16:

Proposition 4.17. Suppose $C$ is compact coisotropic submanifold. Then the following implication holds for the local symplectic model of $C$ :

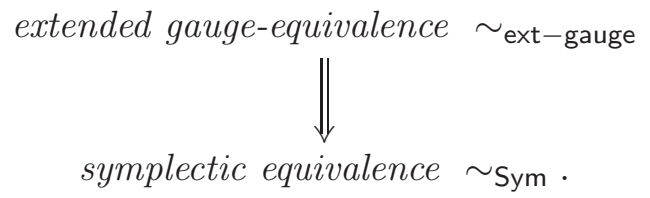

Combining the two previous propositions, we obtain the main result of this section:

Theorem 4.18. Let $C$ be a compact coisotropic submanifold with local symplectic model $(U, \Omega)$. The equivalence relations on

$$
\operatorname{Def}_{U}(C):=\{s \in \Gamma(E): s \text { is coisotropic and } \operatorname{graph}(s) \subset U\}
$$

given by 
- symplectic equivalence $\sim_{\mathrm{Sym}}($ Definition 4.1$)$ and

- extended gauge-equivalence $\sim_{\text {ext-gauge }}$ (Definition 4.12, see also Proposition 4.14) coincide.

As a consequence we have:

Theorem 4.19. Let $C$ be a compact coisotropic submanifold with local symplectic model $(U, \Omega)$. The bijection

descends to a bijection

$$
\operatorname{Def}_{U}(C) \cong \mathrm{MC}_{U}(\Gamma(\wedge E)[1])
$$

$$
\mathcal{M}_{U}^{\mathrm{Sym}}(C):=\operatorname{Def}_{U}(C) / \sim_{\text {Sym }} \cong \mathrm{MC}_{U}(\Gamma(\wedge E)[1]) / \sim_{\text {ext-gauge }} .
$$

4.5. Comparison with Hamiltonian equivalence. In this note we considered both Hamiltonian equivalence (Definition 3.1) and symplectic equivalence (Definition 4.1) of coisotropic submanifolds. Here we summarize some results of Ruan [13] about the relation between these two kinds of equivalence. Ruan considers a restricted class of coisotropic submanifolds, which he calls integral.

Definition 4.20. A coisotropic submanifold $C$ is integral if the leaves of its characteristic foliation $\mathfrak{F}$ are all compact and the set of leaves $S$ admits a smooth structure such that the natural map $C \rightarrow S$ is a submersion. (In other words: $C \rightarrow S$ is a smooth fibre bundle with compact fibres.)

\section{Remark 4.21.}

(1) As Ruan noticed in 13, being integral is not preserved under small deformations inside the space of coisotropic submanifolds. In the following, we restrict attention to the space of coisotropic sections which are integral, and denote them by $\operatorname{Def}_{U}^{\mathrm{int}}(C)$.

(2) Recall that every fibre bundle $p: C \rightarrow S$ with compact fibres $S$ inherits a local system $\mathbf{H}$, given by the fibrewise cohomology, i.e.

$$
\mathbf{H}_{s}:=H^{\bullet}\left(p^{-1}(s), \mathbb{R}\right),
$$

equipped with the Gauss-Manin connection. The cohomology $H^{\bullet}(S, \mathbf{H})$ is the second sheet of the Leray-Serre spectral sequence associated to $p: C \rightarrow S$, which converges to the cohomology of $C$. We will focus on $\mathbf{H}_{s}^{1}:=H^{1}\left(p^{-1}(s), \mathbb{R}\right)$. Observe that the differential $d_{2}$ of the second sheet gives a natural linear map

$$
d_{2}: H^{0}\left(S, \mathbf{H}^{1}\right) \rightarrow H^{2}\left(S, \mathbf{H}^{0}\right) .
$$

Notice that the former group is the space of global, flat sections of the vector bundle $\mathbf{H}^{1}$ over $S$. Since the fibres of $p$ are connected, the latter group is just $H^{2}(S, \mathbb{R})$.

In [13, Theorem 1] Ruan establishes the following result:

Theorem 4.22. Let $C$ be an integral coisotropic submanifold.

(1) There is an open embedding

$$
\operatorname{Def}_{U}^{\text {int }}(C) / \sim_{\text {Ham }} \hookrightarrow H^{0}\left(S, \mathbf{H}^{1}\right) .
$$

(2) The image of the equivalence class of $C$ with respect to symplectic equivalence $\sim_{\text {Sym }}$ under the map $\operatorname{Def}_{U}^{\text {int }}(C) \rightarrow \operatorname{Def}_{U}^{\text {int }}(C) / \sim_{\text {Ham }}$ is given nearby $C$ as the kernel of $d_{2}: H^{0}\left(S, \mathbf{H}^{1}\right) \rightarrow H^{2}(S, \mathbb{R})$. 
Below we reproduce an example from [13]:

Example 4.23. Consider the unit sphere $C=S^{3}$ in $\mathbb{R}^{4}$, with the canonical symplectic form. The characteristic leaves of $S^{3}$ are circles, and $p: S^{3} \rightarrow S=S^{2}$ is the Hopf fibration. $\mathbf{H}^{1}$ is a trivial rank 1 vector bundle over $S^{2}$, so $H^{0}\left(S, \mathbf{H}^{1}\right) \cong \mathbb{R}$, one generator being represented by a connection 1 -form on the Hopf fibration. The map $H^{0}\left(S, \mathbf{H}^{1}\right) \rightarrow H^{2}(S, \mathbb{R}) \cong \mathbb{R}$ is an isomorphism, reflecting the fact that the connection is not flat.

Hence, by Theorem 4.22, not all nearby integral coisotropic deformations of $S^{3}$ are related to $C$ by a symplectomorphism, for instance all spheres of radius $r$ for $r \neq 1$ are not. But those which are, are actually equivalent to $C$ by a Hamiltonian diffeomorphism. The latter statement follows, since $H^{1}(C)=0$ implies that all symplectic vector fields in a tubular neighborhood of $C$ are Hamiltonian.

Another example is:

Example 4.24. Consider the 3 -torus $C=\mathbb{T}^{3}$, which "coordinates" $\theta_{1}, \theta_{2}, \theta_{3}$, as the zero section of $\left(\mathbb{T}^{3} \times \mathbb{R}, d \theta_{1} \wedge d \theta_{2}+d \theta_{3} \wedge d x_{4}\right)$, where $x_{4}$ is the standard coordinate on $\mathbb{R}$. The characteristic leaves are again circles, and $p: \mathbb{T}^{3} \rightarrow S=\mathbb{T}^{2}$ is the trivial fibration. Again, $\mathbf{H}^{1}$ is a trivial rank 1 vector bundle, so $H^{0}\left(S, \mathbf{H}^{1}\right) \cong \mathbb{R}$, one generator being represented by $d \theta_{3}$. The map $H^{0}\left(S, \mathbf{H}^{1}\right) \cong \mathbb{R} \rightarrow H^{2}(S, \mathbb{R}) \cong \mathbb{R}$ is the zero map, reflecting the fact that $d \theta_{3}$ is a closed 1 -form.

We conclude that all nearby integral coisotropic deformations of $C$ are related to $C$ by a symplectomorphism, but not all of them are related to $C$ by a Hamiltonian diffeomorphism. For instance, the 3 -tori given by $\left\{x_{4}=c\right\}$ for constants $c \neq 0$ are not. Notice that the latter

statement is in accordance with the fact that $\mathcal{M}_{U}^{\text {Sym }}(C) \neq \mathcal{M}_{U}^{\mathrm{Ham}}(C)$, which is a consequence of Remark 4.6 since the map $r: H^{1}(C) \rightarrow H_{\mathfrak{F}}^{1}(C)$ has one-dimensional image.

\section{The transversally integrable CASE}

In this section we consider coisotropic submanifolds $C$ that admit a foliation that is complementary to the characteristic foliation:

Definition 5.1. A coisotropic submanifold $C$ of $(M, \omega)$ is called transversally integrable if the kernel $K$ of the pre-symplectic structure $\omega_{C}$ admits a complementary subbundle $G$ which is involutive.

Remark 5.2. A transversally integrable coisotropic submanifold $C$ comes equipped with two foliations: the characteristic foliation $\mathfrak{F}$, given by the maximal leaves of $K$, and another foliation, given by the maximal leaves of $G$. Since $K$ is the kernel of the pre-symplectic structure on $C$, the leaves of $G \cong T C / K$ inherit a symplectic structure.

The assumption of transversal integrability leads to many simplifications. We recover a result by $\mathrm{Oh}$ and Park [12] that says that the $L_{\infty}[1]$-algebra associated to a transversally integrable $C$ is a differential graded Lie algebra (Proposition 5.3). Moreover, we give a formula for the coisotropic section generated by moving the zero section by a basic Hamiltonian flow (Proposition 5.12). 
5.1. Oh and Park's $L_{\infty}[1]$-algebra. Let $C$ be a coisotropic submanifold and $(U, \Omega)$ be the local symplectic model of $C$ as in Section 1. As seen there, the normal model is a neighborhood of the zero section in a vector bundle $E \rightarrow C$, so it comes equipped with a surjective submersion $\pi: U \rightarrow C$.

The following proposition was already proven in [12, Equation (9.17)] (see also Theorem 9.3 there). We provide an alternative proof here.

Proposition 5.3. Let $C$ be a coisotropic submanifold, and assume there exists an involutive complement $G$ to $K=\operatorname{ker}\left(\omega_{C}\right)$. Then the $L_{\infty}[1]$-algebra structure on $\Gamma(\wedge E)[1], E=K^{*}$, associated to $C$ as in Subsection 2.3 correspond 6 to a differential graded Lie algebra.

Proof. The structure maps $\lambda_{r}$ of the $L_{\infty}$ [1]-algebra from Subsection 2.3 are derivations in each argument. Consequently they can be evaluated locally. Moreover, the derivation property and a degree-count using the fact that $\Pi$ is a bivector field show that it suffices to evaluate them on tuples of the form

$$
\left(f, g, s_{1}, \ldots, s_{r-2}\right), \quad\left(f, s_{1}, \ldots, s_{r-1}\right) \quad \text { and } \quad\left(s_{1}, \ldots, s_{r}\right)
$$

with $f, g \in \mathcal{C}^{\infty}(C)$ and $s_{i} \in \Gamma(E)$, seen as vertical vector fields on $E$, in order to determine them completely.

We now compute the multibracket $\lambda_{k}$ of $\mathrm{Oh}$ and Park's $L_{\infty}[1]$-algebra structure on $\Gamma(\wedge E)[1]$ in local coordinates. As we already noticed, the leaves of the involutive subbundle $G$ complementary to $K$ are symplectic. Choose coordinates $q_{1}, \ldots, q_{n-k}, y_{1}, \ldots, y_{2 k}$ on $C$ adapted to the foliations integrating $K$ and $G$, respectively. That is, $K$ is spanned by the $\frac{\partial}{\partial q}$ 's and $G$ is spanned by the $\frac{\partial}{\partial y}$ 's. Add conjugate coordinates $p_{1}, \ldots, p_{n-k}, u_{1}, \ldots, u_{2 k}$ to obtain a coordinate system on $T^{*} C$. The subbundle $G^{\circ} \subset T^{*} C$ is locally given by $\left\{u_{1}=\cdots=u_{2 k}=0\right\}$. Hence the symplectic form on $E=K^{*} \cong G^{\circ}$ (see Section 1) reads

$$
\Omega=\sum_{i=1}^{n-k} d q_{i} \wedge d p_{i}+\pi^{*} \omega_{C} .
$$

Notice that, in coordinates, $\omega_{C}$ has the form $\sum h_{j l} d y_{j} \wedge d y_{l}$ for some functions $h_{j l}$ on $C$. Notice further that $\Omega$ (and therefore the Poisson bivector field $\Pi$ obtained by inverting $\Omega$ ) are invariant under all of the vertical vector fields $\frac{\partial}{\partial p_{1}}, \ldots, \frac{\partial}{\partial p_{n-k}}$. The structure maps $\lambda_{r}$ are determined by their evaluation on tuples of the form

$$
\left(f, g, \frac{\partial}{\partial q_{i_{1}}}, \ldots, \frac{\partial}{\partial q_{i_{r-2}}}\right), \quad\left(f, \frac{\partial}{\partial q_{i_{1}}}, \ldots, \frac{\partial}{\partial q_{i_{r-1}}}\right) \quad \text { and } \quad\left(\frac{\partial}{\partial q_{i_{1}}}, \ldots, \frac{\partial}{\partial q_{i_{r}}}\right) .
$$

Consider the term on the right-hand side of Equation (2) in Subsection 2.3 before applying the projection $P$, that is,

$$
[[\ldots[\Pi,-],-] \ldots],-] .
$$

As we argued above, it suffices to evaluate this expression on tuples consisting of functions on $C$ and vertical vector fields $\frac{\partial}{\partial q_{i}}$. Since $\pi^{*} f$ and $\Omega$ is invariant under any of the vertical vector fields, $\frac{\partial}{\partial q_{i}}$, the structure map $\lambda_{r}$ vanish whenever we evaluate it on a tuple that contains a $\frac{\partial}{\partial q_{i}}$. Hence, only $\lambda_{1}$ and $\lambda_{2}$ can be non-zero.

\footnotetext{
${ }^{6}$ That is, the $L_{\infty}$-algebra obtained after applying the degree shift operator [ -1$]$ is a differential graded Lie algebra, i.e. the structure maps $\lambda_{k}$ vanish for $k>2$.
} 


\section{Remark 5.4.}

(1) The non-trivial structure maps of the differential graded Lie algebra associated to a transversally integrable coisotropic submanifold are given by

$$
\lambda_{1}(f)=P\left(X_{\pi^{*} f}\right) \text { and } \lambda_{2}(f, g)=-\{f, g\}^{G},
$$

the fact that $\lambda_{1}$ and $\lambda_{2}$ annihilate the coordinate vector fields $\frac{\partial}{\partial q}$ associated to adapted coordinates on $C$, and the derivation rule. Here, $\{\cdot, \cdot\}^{G}$ denotes the leafwise Poisson structure associated to the symplectic foliation integrating $G \overline{7}$

(2) The $L_{\infty}$ [1]-algebra we associated to a coisotropic submanifold $C$ depends on the choice of a tubular neighborhood $U$, as well as on the choice of a subbundle $G$ complementary to the kernel $K$ of the pre-symplectic structure. Theorem 4.3 of [3] asserts that different choices of these data lead to isomorphic $L_{\infty}$ [1]-algebras. Consequently Proposition 5.3 guarantees that in case an involutive transversal distribution exists, every $L_{\infty}$ [1]-algebra associated to $C$ is isomorphic to a differential graded Lie algebra.

5.2. Hamiltonian equivalences. We want to be more explicit about lifting constructions from a coisotropic submanifold $C$ to its local symplectic model $(U, \Omega)$. To this end, the concept of a partial Ehresmann connection will be of great importance.

Definition 5.5. Let $K$ be an involutive distribution on $C$. Suppose $\pi: U \rightarrow C$ is a surjective submersion. A partial Ehresmann connection on $U$ is a choice of a complementary subbundle $G$ to $K$ and a subbundle $G^{\sharp}$ of $T U$ such that the differential of $d_{x} \pi$ at $x \in U$ maps $G_{x}^{\sharp}$ isomorphically onto $G_{\pi(x)}$.

Remark 5.6. We notice that the last condition implies that $G^{\sharp}$ is complementary to $(d \pi)^{-1}(K)$.

Lemma 5.7. Let $C$ be a coisotropic submanifold with local symplectic model $(U, \Omega)$. Suppose $G$ is the subbundle complementary to the kernel $K$ of the pre-symplectic form $\omega_{C}$ which was chosen in the construction of $(U, \Omega)$.

(1) The subbundles $(d \pi)^{-1} G$ and $V=\operatorname{ker}(d \pi)$ of $T U$ are symplectically orthogonal to each other.

(2) The bundle

$$
G^{\sharp}:=\left((d \pi)^{-1}(K)\right)^{\perp}
$$

defines a partial Ehresmann connection on $U$.

Proof. We take $\xi \in \operatorname{ker}\left(d_{x} \pi\right)$ and $v \in\left(d_{x} \pi\right)^{-1}(G)$. Plugging the two vectors into the symplectic form $\omega$ yields

$$
\Omega_{x}(\xi, v)=\omega_{C}(d \pi(\xi), d \pi(v))+\omega_{T^{*} C}\left(d_{x} j(\xi), d_{x} j(v)\right)=0 .
$$

Since the ranks of the two subbundles add up to the rank of $T U$, the first claim follows.

Concerning (2), the inclusion $\operatorname{ker}\left(d_{x} \pi\right) \subset\left(d_{x} \pi\right)^{-1}(K)$ implies

$$
\left(\left(d_{x} \pi\right)^{-1}(K)\right)^{\perp} \subset\left(\operatorname{ker}\left(d_{x} \pi\right)\right)^{\perp}=\left(d_{x} \pi\right)^{-1}(G),
$$

\footnotetext{
${ }^{7}$ The additional minus sign in $\lambda_{2}$ is a consequence of the fact that we work in $\Gamma(\wedge E)[1]$, i.e. that we shift all the degrees down be one. In particular, functions have degree -1 after this shift.
} 
i.e. $G^{\sharp}$ maps indeed onto $G$ under $d \pi$. To check that the map is an isomorphism, it suffices to check that the dimensions match, which is straight-forward.

Remark 5.8. The partial Ehresmann connection $G^{\sharp}$ was first considered in [12], see Equation (6.3) there. Observe that $G^{\sharp}$ is usually not linear, i.e. not compatible with the linear structure on $E \supset U$.

A partial Ehresmann connection $G^{\sharp}$ is called flat if it is an involutive subbundle of $T U$. This condition can be restated as follows: $G^{\sharp}$ gives rise to a map

$$
\Gamma(G) \rightarrow \mathcal{X}(U), \quad X \mapsto X^{\text {hor }},
$$

where $X^{\text {hor }}$ is uniquely determined by the condition $d_{x} \pi\left(\left.X^{\text {hor }}\right|_{x}\right)=X_{\pi(x)}$ for all $x \in U$. Flatness of $G^{\sharp}$ is equivalent to the requirements that $G$ is involutive and that the map $X \mapsto X^{\text {hor }}$ is compatible with the Lie bracket of vector fields.

Proposition 5.9. Let $C$ be a coisotropic submanifold that is transversally integrable, with $G$ an involutive transversal distribution. Let $(U, \Omega)$ be the corresponding local symplectic model.

(1) The partial connection $G^{\sharp}$ on $U \subset E$ is linear and flat.

(2) For all $f \in \mathcal{C}^{\infty}(C)$, we have

$$
X_{\pi^{*} f}=P\left(X_{\pi^{*} f}\right)+\left(X_{f}^{G}\right)^{\text {hor }}
$$

where:

(i) $P\left(X_{\pi^{*} f}\right) \in \Gamma(E)$ is seen as a vertical vector field on $U \subset E$, constant along the fibres,

(ii) $X_{f}^{G}$ denotes the leafwise Hamiltonian vector field of $f$ with respect to the symplectic foliation integrating $G$ and

(iii) $\left(X_{f}^{G}\right)^{\text {hor }}$ denotes the horizontal lift of $X_{f}^{G}$ with respect to the partial Ehresmann connection $G^{\sharp}$.

Proof. Choose coordinates $y_{1}, \ldots, y_{2 k}, q_{1}, \ldots, q_{n-k}, p_{1}, \ldots, p_{n-k}$ on $U$ as in the proof of Proposition 5.3 .

(1) Equation (6) shows that at every point $x \in U, \Omega_{x}$ is the sum of two symplectic forms, one defined on the subspace spanned by the $\frac{\partial}{\partial p}$ and $\frac{\partial}{\partial q}$ 's, the other one defined on the subspace spanned by the $\frac{\partial}{\partial y}$ 's. As $\left((d \pi)^{-1} E\right)^{\circ}$ is spanned by the $d y$ 's, we obtain

$$
G^{\sharp}=\operatorname{span}\left\{\frac{\partial}{\partial y_{1}}, \ldots, \frac{\partial}{\partial y_{2 k}}\right\} .
$$

In other words, in the trivialization of the vector bundle $E=K^{*}$ given by the chosen coordinates, $G^{\sharp}$ is a trivial partial connection.

From this we deduce that the parallel transport with respect to $G^{\sharp}$ along paths contained in a leaf of $G$ is given by linear isomorphisms between the fibres of $E$, showing that the partial connection $G^{\sharp}$ is linear. Second, the linear partial connection $G^{\sharp}$ is flat, since the distribution $G^{\sharp}$ is clearly involutive.

(2) In the above coordinates, by Equation (6), we have

$$
X_{\pi^{*} f}=\left[\Pi, \pi^{*} f\right]=\sum_{i} \frac{\partial f}{\partial q_{i}} \frac{\partial}{\partial p_{i}}+\left(X_{f}^{G}\right)^{\mathrm{hor}},
$$


where for the horizontal component we used (17). Its vertical component is invariant under each of the vertical vector fields $\frac{\partial}{\partial p_{1}}, \ldots, \frac{\partial}{\partial p_{n-k}}$, therefore it agrees with the vertical component at $\pi(x) \in C$, which is $P\left(X_{\pi^{*} f}\right)$.

Remark 5.10. Our next aim is to explicitly describe the sections of $E$ which are Hamiltonian equivalent to the zero section $\iota: C \rightarrow E$. If $C$ is compact, we can replace Hamiltonian equivalence by base Hamiltonian equivalence, see Definition 3.17 and Propositions 3.18 and 3.19. Recall that this means that we have to consider the time one flow of a time-dependent vector field $X_{\pi^{*} f_{t}}$ where $f_{t} \in \mathcal{C}^{\infty}(C)$. Such vector fields are not vertical in general, hence solving explicitly the ODE to find their flow is not easy. We are able to do so when $G$ is involutive, making use of the following result:

Lemma 5.11. Let $A \rightarrow M$ be a vector bundle with a linear connection $\nabla$. Let $\left(X_{t}\right)_{t \in[0,1]}$ be a one-parameter family of vector fields on $M$, and $\left(\alpha_{t}\right)_{t \in[0,1]}$ a one-parameter family of sections of $A$. Consider the one-parameter family of vector fields on $A$ given by

$$
\alpha_{t}+\left(X_{t}\right)^{\text {hor }}
$$

where $\alpha_{t}$ is viewed as a vertical vector field which is constant along the fibres of $A$, and $\left(X_{t}\right)^{\text {hor }}$ is the horizontal lift of $X_{t}$ with respect to the connection $\nabla$. The integral curve of $\alpha_{t}+\left(X_{t}\right)^{\text {hor }}$ starting at $q \in C$ is given by

$$
s(t)=\int_{0}^{t} \gamma(t) \backslash \backslash\left[\left.\alpha_{\tau}\right|_{\gamma(\tau)}\right] d \tau \in A_{\gamma(t)}
$$

where $\$ denotes the parallel transport with respect to $\nabla$ along the curve $\gamma(t):=\psi_{t}(q)$, and $\psi_{t}: C \rightarrow C$ the flow of $X_{t}$.

Proof. We have $s=A \circ \Delta$, where $\Delta:[0,1] \rightarrow[0,1]^{2}$ is the diagonal map and

$$
A(r, t):=\int_{0}^{r} \gamma(t) \mathbb{\gamma ( \tau )} \backslash\left[\left.\alpha_{\tau}\right|_{\gamma(\tau)}\right] d \tau
$$

Hence $\left.\frac{\partial}{\partial t}\right|_{t_{0}} s(t)=\left.\frac{\partial}{\partial t}\right|_{t_{0}} A\left(t, t_{0}\right)+\left.\frac{\partial}{\partial t}\right|_{t_{0}} A\left(t_{0}, t\right)$ can be written as the sum of two terms, for all $t_{0} \in[0,1]$. The first one is the vertical vector

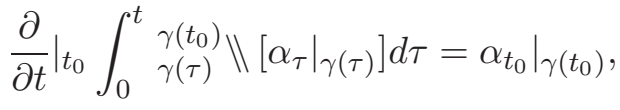

as can be seen noticing that the integrand is a curve in $A_{\gamma\left(t_{0}\right)}$, parametrized by $s$, and applying the fundamental theorem of calculus.

For the second term, we claim that

$$
\left.\frac{\partial}{\partial t}\right|_{t_{0}} \int_{0}^{t_{0}} \begin{aligned}
& \gamma(t) \\
& \gamma(\tau)
\end{aligned} \|\left[\left.\alpha_{\tau}\right|_{\gamma(\tau)}\right] d \tau=\left.\left(X_{t_{0}}\right)^{\text {hor }}\right|_{s\left(t_{0}\right)}
$$

Notice that the integral on the left-hand side of Equation (8) is an element of the fibre of $V$ over $\gamma(t)$, hence applying $\left.\frac{\partial}{\partial t}\right|_{t_{0}}$ we obtain an element of $T_{s\left(t_{0}\right)} A$ that projects to $\left.\frac{\partial}{\partial t}\right|_{t_{0}} \gamma(t)=$ $\left.X_{t_{0}}\right|_{\gamma\left(t_{0}\right)}$ under $\pi$. We now argue that the left-hand side of Equation (8) is a horizontal lift, which would conclude the statement. Let $r, t \in[0,1]$. Under the identification $A_{\gamma(r)} \cong A_{\gamma(t)}$ given by the parallel transport $\begin{aligned} & \gamma(t) \\ & \gamma(r)\end{aligned} \|$, the elements $\underset{\gamma(\tau)}{\gamma(r)} \|\left[\left.\alpha_{\tau}\right|_{\gamma(\tau)}\right]$ and ${ }_{\gamma(\tau)}^{\gamma(t)} \| \backslash\left[\left.\alpha_{\tau}\right|_{\gamma(\tau)}\right]$ agree for every $\tau$. The same holds for the integral from $\tau=0$ to $\tau=t_{0}$ of these elements, since 
parallel transport is a linear isomorphism. Hence the integral on the left-hand side of (8), as $t$ varies, defines a parallel section of $A$ over $\gamma$. Therefore, applying $\left.\frac{\partial}{\partial t}\right|_{t_{0}}$ to it yields an horizontal element of $T_{s\left(t_{0}\right)} A$.

Proposition 5.12. Let $C$ be a compact coisotropic submanifold that is transversally integrable, with $G$ an involutive transversal distribution. Let $(U, \Omega)$ be the corresponding local symplectic model. Take a one-parameter family $\left(f_{t}\right)_{t \in[0,1]} \in \mathcal{C}^{\infty}(C)$, and denote by $\Phi$ the time-1 flow of the time-dependent vector field $\left(X_{\pi^{*} f_{t}}\right)_{t \in[0,1]}$. Then $\Phi(C)$ is the graph of the following section of $U \subset E$ :

$$
p \mapsto \int_{0}^{1} \begin{aligned}
& \sigma(1) \\
& \sigma(t)
\end{aligned} \backslash\left[\left.P\left(X_{\pi^{*} f_{t}}\right)\right|_{\sigma(t)}\right] d t
$$

where $\$ denotes the parallel transport with respect to the partial connection $G^{\sharp}$ along the curve $\sigma(t):=\psi_{t}\left(\left(\psi_{1}\right)^{-1} p\right)$, for $\psi_{t}: C \rightarrow C$ the flow of $X_{f_{t}}^{G}$.

Proof. By Proposition [5.9, $G^{\sharp}$ is a partial linear connection on $U \subset E$, and $X_{\pi^{*} f_{t}}=$ $P\left(X_{\pi^{*} f_{t}}\right)+\left(X_{f_{t}}^{G}\right)^{\text {hor }}$. We note that, in particular, this vector field covers $X_{f_{t}}^{G}$, which is tangent to the leaves of $G$. Fix $p \in C$, and let $L \subset C$ be the leaf of $G$ through $p$. Consider the vector bundle $\left.E\right|_{L} \rightarrow L$, equipped with the linear connection obtained by restricting $G^{\sharp}$. We apply Lemma 5.11 to the one-parameter family of vector fields $\left.\left(X_{f_{t}}^{G}\right)\right|_{L}$ and to the one-parameter family of sections $\left.P\left(X_{\pi^{*} f_{t}}\right)\right|_{L}$. Choosing the point $q$ so that $\psi_{1}(q)=p$ and setting $t=1$ finishes the proof.

\section{Remark 5.13.}

(1) We observe that Propositions 5.9 and 5.12 continue to hold for symplectomorphisms, i.e. one obtains explicit formulae for the symplectic vector field associated to a closed 1 -form obtained via pull-back from the base $C$, as well as for the image of $C$ under the flow of such a vector field.

(2) When $C$ is Lagrangian, $U$ is open in the cotangent bundle $T^{*} C$, hence $X_{\pi^{*} f_{t}}$ is a (constant) vertical vector field and $P\left(X_{\pi^{*} f_{t}}\right)=d f_{t}$. Further $G=\{0\}$, so the curve $\sigma$ through $p$ is constant. Therefore we recover the well-known result that $\Phi(C)$ is the graph of the exact one-form $d\left(\int_{0}^{1} f_{t} d t\right)$.

We exemplify the above discussion in the case of $C$ hypersurface, i.e. of co-dimension 1. While all smooth deformations of a co-dimension 1 submanifold are automatically coisotropic, it turns out that the equivalence problem is non-trivial.

Example 5.14. Fix a codimension 1 compact submanifold $C$ of $(M, \omega)$, which we assume to be oriented. The annihilator $T C^{\circ} \cong K$ is a trivial line bundle, so there is $\alpha \in \Omega^{1}(C)$ such that $G:=\operatorname{ker}(\alpha)$ satisfies $G \oplus E=T C$. As usual $K$ is the characteristic distribution of $C$, i.e., $K:=\operatorname{ker}\left(\omega_{C}\right)$. We assume that $d \alpha=0$, which in particular implies that $G$ is involutive. By [11, Exercise 3.36] a tubular neighborhood of $C$ in $M$ is symplectomorphic to

$$
(U, \Omega):=\left(C \times I, \pi^{*} \omega_{C}-d u \wedge \pi^{*} \alpha\right),
$$

where $I$ is an open interval containing $0, u$ the standard coordinate on $I$, and $\pi: C \times I \rightarrow C$ is the projection. In the following we denote by $\hat{\xi}$ the unique vector field on $C$ lying in $K$ such that $\alpha(\hat{\xi})=1$. 
Take a one-parameter family $f_{t} \in \mathcal{C}^{\infty}(C)$, and denote by $\Phi$ the time- 1 flow of the vector field $\left(X_{\pi^{*}} f_{t}\right)_{t \in[0,1]}$. Then $\Phi(C)$ is the graph of

$$
s: C \rightarrow \mathbb{R}, \quad s(p)=-\left.\int_{0}^{1} \hat{\xi}\left(f_{t}\right)\right|_{\psi_{t}\left(\left(\psi_{1}\right)^{-1} p\right)} d t
$$

where $\psi_{\tau}: C \rightarrow C$ is the flow of $\left(X_{f_{t}}^{G}\right)_{t \in[0,1]}$. This follows from Prop. 5.12, since $X_{\pi^{*} f}=$ $-\hat{\xi}\left(f_{t}\right) \frac{\partial}{\partial u}+X_{f}^{G}$ at points of $C$, and $G^{\sharp}$ is the trivial partial connection by Equation (7).

\section{Appendix A. Fibrewise entire Poisson structures}

In the body of the paper we worked with symplectic structures, but most of the results extend to fibrewise entire Poisson structures, as defined in [16]. More precisely, we assume the following set-up in this appendix:

$U$ is a tubular neighborhood of the zero section in a vector bundle $E \rightarrow C$, $\Pi$ is a fibrewise entire Poisson structure on $U$, such that the zero section $C$ is coisotropic.

Apart from the symplectic case, an interesting example is when $E$ is the dual of a Lie algebroid $(A, \rho,[\cdot, \cdot])$ and $\Pi$ the canonical Poisson structure defined there. As described in [6. Remark 4.5], one can furthermore enhance this example as follows: given a Lie subalgebroid $B \hookrightarrow A$, its fibrewise annihilator $B^{\circ} \subset E$ is a coisotropic submanifold. If the Lie algebroid structure varies in an analytic fashion along the normal bundle to the base of $B$, one can find a tubular neighborhood of $B^{\circ} \subset E$ such that $\pi$ becomes fibrewise entire.

The results obtained in Sections 2 and 3 continue to hold if one replaces the Lie algebroid $K=\operatorname{ker} \omega_{C}$, which is no longer defined, with $(T C)^{\circ}=E^{*}$, the Lie algebroid associated to the coisotropic submanifold $C$ of $(U, \Pi)$. Consequently, one has to replace the foliated de Rham complex $\Omega_{\mathfrak{F}}(C)$ with the complex $(\Gamma(\wedge E), P([\Pi,-])$. Many of the proofs in the main body of the article are already formulated in this setting, and some of them actually simplify in the fibrewise entire Poisson case (for instance Corrollary 3.5).

Concerning Section 4, we replace "symplectomorphisms" in Def. 4.1 by "Poisson diffeomorphisms", and denote the resulting moduli space by $\mathcal{M}_{U}^{\text {Pois }}(C)$. The description of the tangent space at zero to this moduli space is now characterized in terms of Lie algebroid cohomology, as we explain in the next remark:

Remark A.1. The tangent space at zero to $\mathcal{M}_{U}^{\text {Pois }}(C)$ is isomorphic to the quotient

$$
\frac{\{s \in \Gamma(E): P([\Pi, s])=0\}}{\{P(Y): Y \text { is a Poisson vector field on } U\}} \text {. }
$$

Indeed the numerator is the formal tangent space to $\operatorname{Def}_{U}(C)$ by the proof of Proposition 2.4. For the denominator, we argue as follows: if $Y_{t}$ is a one-parameter family of Poisson vector fields on $U$, and $s_{t} \in \Gamma(U)$ is such that the graph of $s_{t}$ is the image of the zero section under the time- $t$ flow of $Y_{t}$, then $\left.\frac{\partial s_{t}}{\partial t}\right|_{t=0}=P\left(Y_{0}\right)$ by Lemma 3.13, and notice that this argument can be reversed. 
We can describe (9) as the cokernel of a certain map in cohomology, by finding the analog of Equation (5) that holds in the Poisson case. We have a map

$$
P: \chi^{\bullet}(U)=\Gamma\left(\wedge T^{*} U\right) \rightarrow \Gamma(\wedge E)
$$

between the complexes of "forms" for the Lie algebroid $T^{*} U$ on one side (the cotangent Lie algebroid of the Poisson manifold $(U, \Pi)$ ) and the Lie algebroid $E=(T C)^{\circ}$ on the other (the Lie algebroid of the coisotropic submanifold $C$ ). The differentials are preserved, since for all $Y \in \chi^{\bullet}(U)$ we have $P[\Pi, Y]=P[\Pi, P Y]$, as a consequence of the relation $P[x, y]=P[P x, y]+P[x, P y]$ that holds in the general setting of Voronov's derived brackets. Another way to see this is to notice that $P$ is the cochain map associated to a Lie algebroid morphism, namely the inclusion of $(T C)^{\circ}$ in $T^{*} U$.

Hence we obtain a map in cohomology

$$
P: H_{L A}\left(T^{*} U\right)=H_{\Pi}(U) \rightarrow H_{L A}\left((T C)^{\circ}\right)
$$

between the Lie algebroid cohomology of $T^{*} U$ (i.e. the Poisson cohomology of $(U, \Pi)$ ) and the Lie algebroid cohomology of $(T C)^{\circ}$. Its cokernel agrees with (9) by a linear algebra argument as in Remark 4.6, which uses the fact that for any function $F$ on $U$ we have $P[\Pi, F]=P\left[\Pi,\left.F\right|_{C}\right]$.

Let us finally point out the place where the case of fibrewise entire Poisson structures deviates most seriously from the symplectic case: it is no longer obvious that Poisson vector fields can be replaced by fibrewise entire ones. Therefore we cannot establish Proposition 4.16, and consequently neither Theorem 4.18 nor Theorem 4.19 carry over.

\section{REFERENCES}

[1] A. S. Cattaneo and G. Felder. Coisotropic submanifolds in Poisson geometry and branes in the Poisson sigma model. Lett. Math. Phys., 69:157-175, 2004.

[2] A. S. Cattaneo and G. Felder. Relative formality theorem and quantisation of coisotropic submanifolds. Adv. in Math., 208:521-548, 2007.

[3] A. S. Cattaneo and F. Schätz. Equivalences of higher derived brackets. J. Pure Appl. Algebra, 212(11):2450-2460, 2008.

[4] Y. Frégier and M. Zambon. $L_{\infty}$-algebras governing simultaneous deformations via derived brackets. 02 2012, ArXiv: 1202.2896.

[5] M. J. Gotay. On coisotropic imbeddings of presymplectic manifolds. Proc. Amer. Math. Soc., 84(1):111$114,1982$.

[6] X. Ji. Simultaneous deformations of a Lie algebroid and its Lie subalgebroid. J. Geom. Phys., 84:8-29, 2014.

[7] A. Kapustin and D. Orlov. Remarks on A-branes, mirror symmetry, and the Fukaya category. J. Geom. Phys., 48(1):84-99, 2003.

[8] T. Lada and J. Stasheff. Introduction to SH Lie algebras for physicists. Internat. J. Theoret. Phys., 32(7):1087-1103, 1993.

[9] H. V. Lê and Y.-G. Oh. Deformations of coisotropic submanifolds in locally conformal symplectic manifolds. 08 2012, ArXiv:1208.3590.

[10] H. V. Lê, Y.-G. Oh, A. G. Tortorella, and L. Vitagliano. Deformations of Coisotropic Submanifolds in Abstract Jacobi Manifolds. 10 2014, ArXiv:1410.8446.

[11] D. McDuff and D. Salamon. Introduction to symplectic topology. Oxford Mathematical Monographs. The Clarendon Press, Oxford University Press, New York, second edition, 1998.

[12] Y.-G. Oh and J.-S. Park. Deformations of coisotropic submanifolds and strong homotopy Lie algebroids. Invent. Math., 161(2):287-360, 2005. 
[13] W.-D. Ruan. Deformation of integral coisotropic submanifolds in symplectic manifolds. J. Symplectic Geom., 3(2):161-169, 2005.

[14] F. Schätz. BFV-complex and higher homotopy structures. Comm. Math. Phys., 286(2):399-443, 2009.

[15] F. Schätz. Moduli of coisotropic sections and the BFV-complex. Asian J. Math., 15(1):71-100, 2011.

[16] F. Schätz and M. Zambon. Deformations of coisotropic submanifolds for fibrewise entire Poisson structures. Lett. Math. Phys., 103(7):777-791, 2013.

[17] J. D. Stahseff. The intrinsic bracket on the deformation complex of an associative algebra. J. Pure Appl. Algebra, 89:231-235, 1993.

[18] T. Voronov. Higher derived brackets and homotopy algebras. J. Pure Appl. Algebra, 202(1-3):133-153, 2005.

[19] T. T. Voronov. Higher derived brackets for arbitrary derivations. In Travaux mathématiques. Fasc. XVI, Trav. Math., XVI, pages 163-186. Univ. Luxemb., Luxembourg, 2005.

[20] M. Zambon. An example of coisotropic submanifolds $C^{1}$-close to a given coisotropic submanifold. Differential Geom. Appl., 26(6):635-637, 2008.

E-mail address: florian.schaetz@gmail.com

Centre for Quantum Geometry of Moduli Spaces, Aarhus University, Ny Munkegade 118, DK-8000 Aarhus C, Denmark.

E-mail address: marco.zambon@uam.es, marco.zambon@icmat.es, marco.zambon@wis.kuleuven.be

Universidad Autónoma de Madrid (Departamento de Matemáticas) and ICMAT(CSiC-UAMUC3M-UCM), Campus de Cantoblanco, 28049 - Madrid, Spain. Current address: Ku Leuven, Department of Mathematics, Celestijnenlaan 200B box 2400, BE-3001 Leuven, Belgium. 